\title{
Noise reduction evaluation of multi-layered viscoelastic infinite cylinder under acoustical wave excitation
}

\author{
M.R. Mofakhami ${ }^{\mathrm{a}}$, H. Hosseini Toudeshky, a, and Sh. Hosseini Hashemi ${ }^{\mathrm{b}}$ \\ ${ }^{a}$ Aerospace Engineering Department, Amirkabir University of Technology (Tehran Polytechnic), 424 Hafez Avenue, \\ Tehran 158754413, Iran \\ ${ }^{\mathrm{b}}$ School of Mechanical Engineering, Iran University of Science and Technology Narmak, Tehran, Iran
}

Received 10 January 2006

\begin{abstract}
In this paper sound transmission through the multilayered viscoelastic air filled cylinders subjected to the incident acoustic wave is studied using the technique of separation of variables on the basis of linear three dimensional theory of elasticity. The effect of interior acoustic medium on the mode maps (frequency vs geometry) and noise reduction is investigated. The effects of internal absorption and external moving medium on noise reduction are also evaluated. The dynamic viscoelastic properties of the structure are rigorously taken into account with a power law technique that models the viscoelastic damping of the cylinder. A parametric study is also performed for the two layered infinite cylinders to obtain the effect of viscoelastic layer characteristics such as thickness, material type and frequency dependency of viscoelastic properties on the noise reduction. It is shown that using constant and frequency dependent viscoelastic material with high loss factor leads to the uniform noise reduction in the frequency domain. It is also shown that the noise reduction obtained for constant viscoelastic material property is subjected to some errors in the low frequency range with respect to those obtained for the frequency dependent viscoelastic material.

Noise reduction analyses are also performed for the infinite cylinder subjected to the periodic incident wave with uniform and piecewise form.
\end{abstract}

Keywords: Noise reduction, infinite cylinder, viscoelastic material, plane wave

\section{Introduction}

Hollow cylindrical structures are used in many practical systems such as marine structures, payload fairings of the launch vehicles, aircraft fuselages and gas pipe lines. In most of the investigations, the cylindrical structures are considered as an infinite length to eliminate the effect of the end boundary conditions in the solution procedure. Doolittle and Uberal [22] provided a classical normal mode solution for the scattered field corresponding to a plane sound wave incident on an infinite elastic circular cylindrical concentric shell, imbedded in a fluid and enclosing another fluid. White [19] studied the transmission of an acoustic wave through the infinite and finite isotropic cylindrical shells which has been derived to be a strong function of frequency and the angle of incident between the axis of the cylinder and the incoming wave. They used the concept of averaging of energy and energy flow over the space, time and frequency band. In this study the average energy and energy flow were used to characterize the physical phenomenon and the individual details of the modal response were not performed. Junger and Garrelick [18] analyzed the scattering of a plane harmonic wave incident on the rigid sphere and a finite cylinder which partially coated with the finite impedance coating and parallel to the incident wave front, by underlying assumptions of

*Corresponding author. Tel.: +98 216454 3224; Fax: +98 216640 4885; E-mail: Hosseini@aut.ac.ir. 
kirchhoff-type formulations of the cross sections of cylindrical and spherical scatters. Koval [15] used thin-walled cylindrical shell equations of Flügge-Lure-Byrne to study the transmission of the obliquely incident plane wave noise into a pressurized aircraft fuselage subjected to a uniform flow in the external medium. Flax and Neubauer [13] performed theoretical investigations on the reflection of plane waves by a layered cylindrical shell immersed in water. They applied their solution to the vacuumed bodies to calculate the reflected pressures. Flax et al. [14] applied the resonance formalism of nuclear-reaction theory to the problem of sound scattering from submerged elastic bodies (Circular cylinders and spheres). Koval [16] investigated the transmission of airborne noise into an aircraft fuselage and the effects of internal cavity resonances on the sound transmission into a thin cylindrical shell. He used the Flügge equation to model the shell. Again Koval [17] provided a mathematical model for the transmission of an oblique plane wave into a laminated composite shell. He also studied the effect of the fibers orientation of composite shells on the noise attenuation. Dickey et al. [8] investigated scattering resulting from a high-frequency plane-wave incident upon an infinite aluminum circular cylindrical shell immersed in and filled with water by applying the Sommerfeld-Watson transformation to the classical Rayleigh normal-mode series solution. The resulting contour integrals computed by both the saddle point and residue theorem methods. Bao et al. [28] investigated the relationship between the resonances and surface waves in the scattering of an obliquely incident acoustic wave from an infinite elastic solid cylinder using three dimensional solution of the normal modes. Bofilios and Lyrintzis [3] investigated structure borne noise transmission of finite double walled and fiber reinforced cylindrical enclosures due to the stochastic excitations. The shell skins were modeled according to a laminated cylindrical thin shell theory and the solution of governing acoustic-structural equations is obtained via modal decomposition and Galerkin like approach. Skelton and James [5] studied the far field sound radiation from anisotropic multi-layered cylinders. They used Fourier integral transform and energy methods considering the excitations, time harmonic point force, point source and oblique plane wave incident. Cheng [12] studied the acoustic field inside a plate-ended cylindrical shell due to a point force. The governing equations of the plate-ended shell were obtained using variational principle via finding the extremum of Hamilton's function over a subspace of displacement trial function, which was followed by Rayleigh-Ritz procedure. The acoustic field was also obtained from Helmotz equation by the means of Green's function and considering the displacement decomposition of the structure with the equations characterizing the acoustic response. Sastry and Munjal $[9,10]$ investigated the noise transmission into a multilayered infinite cylinder using two and three dimensional elasticity theory in two independent studies. They considered an incident plane wave excitation in their models. They used transfer function matrix to obtain the response of the multilayered elastic cylinder with the same matrix coefficient dimension which used for a single layer cylinder. Sound transmission through a periodically stiffened cylindrical shell was investigated by Lee and Kim [6] using Love equations for the motion of cylindrical shell and the principle of virtual work for the system. Again Lee and Kim [7] studied the sound transmission through a double walled cylindrical shell. The incoming noise idealized as a plane wave and inside the medium as an anechoic cavity. In this study, the classical thin-shell theory was used and the sound transmission was calculated from the interaction between the acoustic wave and the bending waves in the shell, besides the one dimensional sound transmission through the layers. Hasheminejad and Safari $[23,24]$ outlined the sound scattering by a viscoelastic structure immersed in viscous fluid that excited by incident plane wave. They investigated the scattered far field pressure and the form function amplitude for the sphere and cylinder filled by viscous fluid.

In the present paper, sound transmission through multilayered viscoelastic infinite cylinders is investigated. Wave propagation through the infinite cylindrical structure is evaluated using the technique of separation of variables on the basis of linear three-dimensional theory of elasticity. The expression for noise reduction is obtained by using the interfacial continuity of pressure and radial acceleration and appropriate acoustic impedance on the inner and outer surfaces. The material properties are considered as viscoelastic frequency dependent type. The analyses are performed for several cases to study the effect of various parameters such as, thickness and type of viscoelastic material in two layered cylinders, angle of incident wave and the motion of the external acoustic medium on the noise transmission. Finally, the results obtained from a part of infinite cylinder exposed to a uniform acoustic wave which is periodically masked from the incident wave are compared with those obtained from the infinite cylinder subjected to a plane wave excitation. Also the analysis of sound transmission of infinite cylinders subjected to a periodic wave excitation using the three dimensional theory of elasticity and considering frequency dependent viscoelastic material has not been done to the authors' knowledge yet. 


\section{Modeling of viscoelastic material}

Absorbers, barriers, mufflers and silencers are used in different configurations to reduce structure-borne vibrations and noises. In most cases, vibrations are isolated or dissipated using isolator or damping materials. Damping controls the steady-state resonant response and attenuates traveling waves in the structure. Material damping is a way of modeling damping in noise reduction analysis. Viscoelastic material exhibits characteristics of both a viscous fluid and an elastic solid. The degree to which a material behaves either viscously or elastically depends mainly on the temperature and rate of loading (frequency). The dynamic properties (shear modulus, extensional modulus, and etc.) of linear viscoelastic materials can be represented by the complex modulus [4]. The viscoelastic materials such as rubbers, elastomers, rigid plastics and polymeric foams, are used intensively for sound and vibration control. Vibration and acoustic problems are usually solved in the frequency domain where the dynamic properties can effectively be characterized by the concept of complex modulus of elasticity. According to this concept the shear and Young's moduli of elasticity and also the Poisson's ratio of the material under vibration, whether isotropic or anisotropic, can be considered as a complex valued function of frequency in the linear range. Recently, there has been an increasing interest in determining the complex Poisson's ratio especially for acoustic materials; therefore, the related measurements concern the audio range or a part of it. It is frequently concluded that the dynamic Poisson's ratio is independent of frequency and the relevant loss factor is nearly zero as an outcome of measurements which made in a narrow frequency range $[21,25,26]$. The damping and dynamic elastic properties of materials play fundamental roles in different fields of acoustical applications. Experimental studies often show that damping and dynamic elastic properties of various solid materials increase with frequency over a finite bandwidth. In addition, a small increase of damping over a finite frequency range has been experienced in stiff structural materials, such as metals in which the damping is usually very low [11]. Acoustical and vibrational analysis requires a mathematical form of the frequency dependencies of dynamic properties. Fractional derivative models offer a powerful tool to describe variations of the dynamic properties over a narrow or wide frequency range without causality problem. Nevertheless, fractional calculus is not mathematically simple and direct because it is defined in the time domain. This relation has to be transformed into the frequency domain and behavior compared with the experimental results to validate the suitability of the fractional model. The method used in this paper is relatively simple and describes the material behavior directly in the frequency domain. The method is based on the experimental observation that the increase of damping in various solid materials appears to follow a simple frequency power law over a finite bandwidth [27]. Since damping and dynamic elastic properties of solid materials in acoustics and structural dynamics are usually characterized through the concept of complex modulus of elasticity, the shear modulus $\bar{G}$ is defined as:

$$
\bar{G}(i \omega)=G_{d}(\omega)+i G_{l}(\omega)=G_{d}(\omega)\left[1+i \eta_{G}(\omega)\right]
$$

where $\omega$ is circular frequency, $G_{d}$ is the dynamic modulus of elasticity, $G_{l}$ is the loss modulus and $\eta_{G}$ is the loss factor defined as:

$$
\eta_{G}(\omega)=\frac{G_{l}(\omega)}{G_{d}(\omega)}
$$

The dynamic properties of materials used for acoustic purposes are usually measured in the audio range over the one to two decade bandwidth frequency. The experimental data often show that the damping properties of these materials increase with increasing the frequency. The increase of the loss modulus may obey a simple frequency power law defined by:

$$
G_{l}(\omega)=c \omega^{\alpha}
$$

where $\alpha$ and $c$ are constants and $c>0$ and $0<\alpha<1$ in most solids. The interdependence between the dynamic modulus and loss modulus is defined through the Kramers-Kronig $(\mathrm{K}-\mathrm{K})$ dispersion relations. Consequently, the negligence of this relation in fitting the frequency functions to the experimental data, which is very common, may lead to a causality problem. The $\mathrm{K}-\mathrm{K}$ relations are a pair of integral equation whose solution leads to a suitable form of the complex modulus [27]:

$$
G_{d}(\omega)=G_{0}+\cot \left(\frac{\alpha \pi}{2}\right) c \omega^{\alpha}
$$

where $G_{0}$ is the static shear modulus. The above relationships are used to model the frequency dependent behavior of the viscoelastic material. 

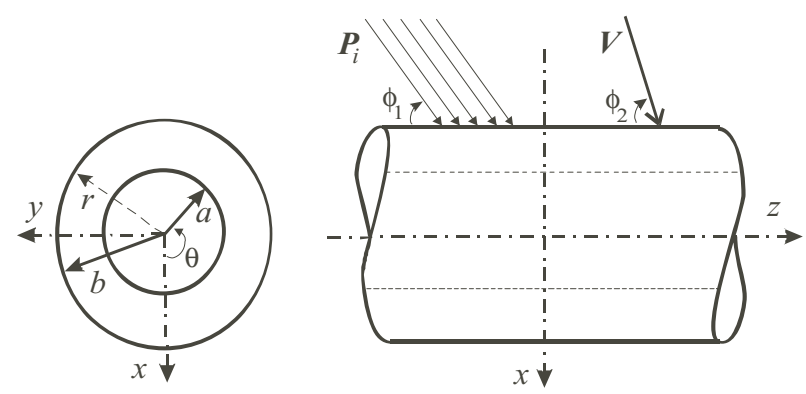

Fig. 1. Infinite cylinder exposed to the incident wave and external medium flow.

\section{Governing equation of hollow infinite cylinder}

In this section the formulation are performed for an isotropic elastic infinite cylinder with the inner and outer radii of $a$ and $b$ as shown in Fig. 1. The corresponding components of displacement vector $\mathbf{u}$ at a point consist of $u_{r}, u_{\theta}$ and $u_{z}$ in the $r, \theta$ and $z$ directions respectively. The displacement equations governing the motion of the isotropic media are:

$$
\rho \frac{\partial^{2} \mathbf{u}}{\partial t^{2}}=\mu \nabla^{2} \mathbf{u}+(\lambda+\mu) \nabla(\nabla \cdot \mathbf{u})
$$

where $\rho$ is the density, $\lambda$ and $\mu$ are the Lame constants and $\nabla^{2}$ is the three-dimensional Laplacian operator. The most general solution of Eq. (5) may be obtained using Helmholtz decomposition technique as:

$$
\mathbf{u}=\nabla \varphi+\nabla \times \mathbf{H}
$$

with

$$
\nabla \cdot \mathbf{H}=F(r, \theta, z, t)
$$

where $\varphi$ and $\mathbf{H}$ are scalar and vector potential functions respectively and $F$ is arbitrary and will be chosen subsequently [1]. Substituting (6) into the (5) results in the following wave equations:

$$
C_{1}^{2} \nabla^{2} \varphi=\frac{\partial^{2} \varphi}{\partial t^{2}}, \quad C_{2}^{2} \nabla^{2} \mathbf{H}=\frac{\partial^{2} \mathbf{H}}{\partial t^{2}}
$$

where:

$$
C_{1}^{2}=\frac{\lambda+2 \mu}{\rho}, \quad C_{2}^{2}=\frac{\mu}{\rho} .
$$

The constants $C_{1}$ and $C_{2}$ are the propagation velocities of dilatational and distortional waves in an infinite medium, respectively. The following set of general solutions can be obtained employing the technique of separation of variables as follows:

$$
\begin{aligned}
\phi(r, \theta, z, t) & =R_{1}\left(\alpha_{1} r\right) T_{1}(\delta z) E_{1}(n \theta) e^{i \omega t} \\
H_{r}(r, \theta, z, t) & =R_{2}\left(\alpha_{2} r\right) T_{2}(\delta z) E_{2}(n \theta) e^{i \omega t} \\
H_{\theta}(r, \theta, z, t) & =R_{3}\left(\alpha_{2} r\right) T_{3}(\delta z) E_{3}(n \theta) e^{i \omega t} \\
H_{z}(r, \theta, z, t) & =R_{4}\left(\alpha_{2} r\right) T_{4}(\delta z) E_{4}(n \theta) e^{i \omega t}
\end{aligned}
$$

where: 


$$
\begin{aligned}
R_{1}\left(\alpha_{1} r\right) & =F_{1} J_{n}\left(\alpha_{1} r\right)+G_{1} Y_{n}\left(\alpha_{1} r\right) \\
R_{2}\left(\alpha_{2} r\right) & =F_{2} J_{n+1}\left(\alpha_{2} r\right)+G_{2} Y_{n+1}\left(\alpha_{2} r\right)+\frac{n}{\alpha_{2} r}\left[\left(F_{3}-F_{2}\right) J_{n}\left(\alpha_{2} r\right)+\left(G_{3}-G_{2}\right) Y_{n}\left(\alpha_{2} r\right)\right] \\
R_{3}\left(\alpha_{2} r\right) & =F_{3} J_{n+1}\left(\alpha_{2} r\right)+G_{3} Y_{n+1}\left(\alpha_{2} r\right)+\frac{n}{\alpha_{2} r}\left[\left(F_{2}-F_{3}\right) J_{n}\left(\alpha_{2} r\right)+\left(G_{2}-G_{3}\right) Y_{n}\left(\alpha_{2} r\right)\right] \\
R_{4}\left(\alpha_{2} r\right) & =F_{4} J_{n}\left(\alpha_{2} r\right)+G_{4} Y_{n}\left(\alpha_{2} r\right) \\
\alpha_{1}^{2} & =\left(\frac{\omega^{2}}{C_{1}^{2}}\right)-\delta_{,}^{2} \alpha_{2}^{2}=\left(\frac{\omega^{2}}{C_{2}^{2}}\right)-\delta^{2} \\
T_{1}(\delta z) & =T_{2}(\delta z)=T_{4}(\delta z)=\left\{\begin{array}{l}
\cos (\delta z) \\
\sin (\delta z)
\end{array}\right\}, T_{3}(\delta z)=\left\{\begin{array}{l}
-\sin (\delta z) \\
\cos (\delta z)
\end{array}\right\} \\
E_{1}(n \theta) & =E_{3}(n \theta)=\left\{\begin{array}{l}
\cos (n \theta) \\
\sin (n \theta)
\end{array}\right\}, E_{2}(n \theta)=E_{4}(n \theta)=\left\{\begin{array}{c}
\sin (n \theta) \\
\cos (n \theta)
\end{array}\right\}
\end{aligned}
$$

and $J_{n}$ and $Y_{n}$ denote the Bessel functions for real arguments that will be replaced by $I_{n}$ and $K_{n}$ for imaginary arguments, $\delta$ is wave number in the axial direction, $n$ is circumferential wave number, $F_{k}$ and $G_{k}(k=1,2,3,4)$ are the constants and $\omega$ is the circular frequency. Substituting of (11) into (10), and the resultant equation into (6) leads to the following displacement components

$$
\begin{aligned}
u_{r}=\{ & \left(\frac{n}{r} J_{n}\left(\alpha_{1} r\right)-\alpha_{1} J_{n+1}\left(\alpha_{1} r\right)\right) A_{1}+\left(\frac{n}{r} Y_{n}\left(\alpha_{1} r\right)-\alpha_{1} Y_{n+1}\left(\alpha_{1} r\right)\right) B_{1} \\
& +\delta J_{n+1}\left(\alpha_{2} r\right) A_{2}+\delta Y_{n+1}\left(\alpha_{2} r\right) B_{2} \\
& \left.+\frac{n J_{n}\left(\alpha_{2} r\right)}{r} A_{3}+\frac{n Y_{n}\left(\alpha_{2} r\right)}{r} B_{3}\right\}\left\{\begin{array}{l}
\cos (\delta z) \\
\sin (\delta z)
\end{array}\right\}\left\{\begin{array}{l}
\cos (n \theta) \\
\sin (n \theta)
\end{array}\right\} e^{i \omega t} \\
u_{\theta}= & -\frac{n J_{n}\left(\alpha_{1} r\right)}{r} A_{1}-\frac{n Y_{n}\left(\alpha_{1} r\right)}{r} B_{1}+\delta J_{n+1}\left(\alpha_{2} r\right) A_{2}+\delta Y_{n+1}\left(\alpha_{2} r\right) B_{2} \\
& -\left(\frac{n}{r} J_{n}\left(\alpha_{2} r\right)-\alpha_{2} J_{n+1}\left(\alpha_{2} r\right)\right) A_{3} \\
& \left.-\left(\frac{n}{r} Y_{n}\left(\alpha_{2} r\right)-\alpha_{2} Y_{n+1}\left(\alpha_{2} r\right)\right) B_{3}\right\}\left\{\begin{array}{c}
\cos (\delta z) \\
-\sin (\delta z)
\end{array}\right\}\left\{\begin{array}{l}
\sin (n \theta) \\
\cos (n \theta)
\end{array}\right\} e^{i \omega t} \\
u_{z}= & -\left\{\delta J_{n}\left(\alpha_{1} r\right) A_{1}+\delta Y_{n}\left(\alpha_{1} r\right) B_{1}+\alpha_{2} J_{n}\left(\alpha_{2} r\right) A_{2}+\alpha_{2} Y_{n}\left(\alpha_{2} r\right) B_{2}\right\}\left\{\begin{array}{l}
\sin (\delta z) \\
\cos (\delta z)
\end{array}\right\}\left\{\begin{array}{l}
\cos (n \theta) \\
\sin (n \theta)
\end{array}\right\} e^{i \omega t}
\end{aligned}
$$

where:

$$
A_{1}=F_{1}, A_{2}=F_{3}, A_{3}=F_{4}\left\{\begin{array}{l}
1 \\
-1
\end{array}\right\}+\frac{\delta}{\alpha}\left(F_{2}-F_{3}\right), B_{1}=G_{1}, B_{2}=G_{3}, B_{3}=G_{4}\left\{\begin{array}{l}
1 \\
-1
\end{array}\right\}+\frac{\delta}{\alpha}\left(G_{2}-G_{3}\right)
$$

In light of Eqs (12) and (13), two solution forms of symmetric mode and antisymmetric mode are obtained which are presented as upper and lower elements in the curly bracket respectively. Utilizing strain-displacement and stress-strain relations, the relevant stress components can be obtained. According to the procedure of solution derivation it can be seen that six of the coefficients $F_{k}$ and $G_{k}(k=1,2,3,4)$ are independent. Hence, without the loss of generality, the constants $F_{4}$ and $G_{4}$ can be set to zero. Therefore, in acoustical problems the displacement and stress expressions can be combined to express in complex functions in the axial direction. These expressions are performed in Appendix A. The aforementioned displacement and stress fields satisfy the equilibrium equations as they should.

To investigate a multi-layered circular cylinder, a cylinder that consists of a circular inner hollow cylinder bounded by a concentric outer cylinder is considered. With reference to the number of coefficients, $A_{k}, B_{k}$, where $k=1,2,3$ in the stress and displacement components, the following continuity conditions must be applied at the cylinders 
interfaces:

$$
\begin{aligned}
& \left(u_{r}\right)_{l}=\left(u_{r}\right)_{l+1} \quad\left(\sigma_{r r}\right)_{l}=\left(\sigma_{r r}\right)_{l+1} \\
& \left(u_{\theta}\right)_{l}=\left(u_{\theta}\right)_{l+1} \quad\left(\sigma_{r \theta}\right)_{l}=\left(\sigma_{r \theta}\right)_{l+1} \quad \text { at } r=r_{l}, l=1,2, . ., m-1, a<r<b \\
& \left(u_{z}\right)_{l}=\left(u_{z}\right)_{l+1} \quad\left(\sigma_{r z}\right)_{l}=\left(\sigma_{r z}\right)_{l+1}
\end{aligned}
$$

where $l$ denotes the $l$ th layer, $m$ and $r_{l}$ are the number of layers and outer radius of the $l$ th layer respectively. To perform free vibration analysis, the infinite circular cylinder may be considered being traction free on the outer surface and therefore the boundary conditions become:

$$
\sigma_{r r}=\sigma_{r \theta}=\sigma_{r z}=0 \quad \text { at } r=b
$$

Assuming the gas exerts no traction force on the solid surface and by the means of acoustic impedance, the following inner surface boundary conditions can be defined:

$$
\sigma_{r r}+i \omega Z_{a} u_{r}=\sigma_{r \theta}=\sigma_{r z}=0 \text { at } r=a
$$

where $Z_{a}$ is the acoustic impedance of the internal medium at the internal boundary wall which calculated as follows:

$$
Z_{a}=\frac{P_{r}}{u_{a}}=-i \rho_{a 2} C_{a 2} \frac{J_{n}\left(\omega a / C_{a 2}\right)}{J_{n}^{\prime}\left(\omega a / C_{a 2}\right)}
$$

and $P_{r}, u_{a}, \rho_{a 2}$ and $C_{a 2}$ are acoustic pressure, radial particle velocity, density and air sound speed respectively. Concerning boundary conditions (15) and (16), for a single layer cylinder, the six simultaneous equations which are linear and homogeneous in the constants $A_{1}, A_{2}, A_{3}, B_{1}, B_{2}$ and $B_{3}$ are obtained. For a nontrivial solution, the determinant formed from the $A$ and $B$ coefficients should be zero. This condition determines the eigen-frequencies of the system. For the case of a multilayered cylinder considering (14), (15) and (16), the coefficient matrix dimension is $(6 \mathrm{~m} \times 6 \mathrm{~m})$ where $m$ is the number of layers. The natural frequencies obtained from the system equation are resonance frequencies of the coupled system of the structure and internal acoustic medium. The mode maps (frequency vs geometry) of the system are presented later in the numerical results section. To calculate the sound transmission due to the incidence of an external acoustic wave, the boundary Eqs (15) and (16) are changed to provide the appropriate radial boundary conditions at the interfaces which are simply the continuity of radial velocities and stresses and will be performed in the following section.

\section{Acoustics}

\subsection{Acoustic excitation}

In the investigated cases, a plane wave is considered as the acoustical wave excitation. Therefore, to evaluate the sound scattering and transmission, a specific problem of a plane wave incident on a flexible infinite hollow cylinder exposed to a uniform flow on the exterior surface is considered as illustrated in Fig. 1. All waves are considered to have the same dependency on the axial coordinate. The harmonic plane incident wave propagated in the positive $x$ direction can be expressed as:

$$
P_{i}(r, \theta, z, t)=P_{o} e^{i\left(\omega t-k_{x} x-k_{z} z\right)}
$$

where $P_{0}$ is amplitude of the incident wave and

$$
k_{x}=k_{1} \sin \left(\phi_{1}\right), k_{z}=k_{1} \cos \left(\phi_{1}\right)
$$

and

$$
k_{1}=\frac{\omega}{C_{a 1}}
$$

in which $k_{1}$ and $C_{a 1}$ are the wave number and sound speed in the external acoustic medium respectively. Expansion of Eq. (18) in the cylindrical coordinates represented by [20] is: 


$$
P_{i}(r, \theta, z, t)=P_{o} \sum_{n=0}^{\infty} \varepsilon_{n}(-i)^{n} J_{n}\left(k_{r} r\right) \cos (n \theta) e^{i\left(\omega t-k_{z} z\right)}
$$

where $n$ is circumferential wave number, $k_{r}$ is equal to the wave number in the $x$ direction and Neumann factor $\varepsilon_{n}$ are defined as:

$$
\begin{aligned}
& \varepsilon_{n}=1 \text { for } n=0 \\
& \varepsilon_{n}=2 \text { for } n \geqslant 1
\end{aligned}
$$

The expression obtained in this part is used to obtain the exterior acoustic field which is investigated in the following section.

\subsection{Interaction of the acoustic field with the cylindrical structure}

In this section the internal and external acoustic fields' equations are obtained and then from the continuity conditions and acting acoustic pressure relationships, the required equations for the noise reduction evaluation are derived. To calculate the pressure in the exterior field, the pressure field due to the incident wave, $P_{i}$, and scattered wave of the cylinder, $P_{s 1}$, must be superimposed. The pressure in the exterior field due to the scattering of the cylinder which has the density of $\rho_{a 1}$ and sound speed of $C_{a 1}$ satisfy the scalar Helmholtz equation, obtained as:

$$
P_{s 1}(r, \theta, z, t)=\sum_{n=0}^{\infty}\left(P_{J} J_{n}\left(k_{r} r\right)+P_{Y} Y_{n}\left(k_{r} r\right)\right) \cos (n \theta) e^{i\left(\omega t-k_{z} z\right)}
$$

where $P_{J}$ and $P_{Y}$ are the coefficients. Since in the far field the outward scattered wave must take on the form of a plane wave as illustrated in Eq. (18), the relationship between the coefficients are obtained as:

$$
P_{J}=-i P_{Y}
$$

Then Eq. (23) can be written as:

$$
P_{s 1}(r, \theta, z, t)=\sum_{n=0}^{\infty} P_{n}^{s 1} \varepsilon_{n}(-i)^{n} H_{n}^{2}\left(k_{r} r\right) \cos (n \theta) e^{i\left(\omega t-k_{z} z\right)}
$$

where $H_{n}^{2}$ is the second kind Hankel function with the integer order of $n . P_{n}^{s 1}=P_{J}$ is an unknown coefficient which should be determined. The radial boundary conditions at the interfaces which imply the continuity of the radial velocities and stresses should be considered. The continuity condition between the radial pressure gradient and acceleration of the structure namely velocity condition represented as:

$$
\frac{\partial P_{t}}{\partial r}=-\rho_{a} \frac{\partial^{2} u_{r}}{\partial t^{2}} \text { at } r=a, b
$$

and

$$
\sigma_{r r}+P_{t}=0 \text { at } r=a, b
$$

where $P_{t}$ is the total pressure (sum of the incident and scattered waves) acting on the structure surface, $\rho_{a}$ indicates the related medium density, $u_{r}$ and $\sigma_{r r}$ are radial structure displacement and stress at the interfaces of cylinder and acoustic medium respectively. Equation (26) denotes the continuity condition between the radial pressure gradient and acceleration of the structure and Eq. (27) shows the relationship between the ambient pressure and radial structure stress which is due to the acting pressure as a distributed force on the cylinder surfaces. In the case of external boundary conditions in Eqs (26) and (27), $P_{t}$ is defined as $P_{i}+P_{s 1}$. Using the Eq. (A-1) from Appendix A, (21) and (25) in Eq. (26) leads to:

$$
\begin{aligned}
\rho_{a 1} \omega^{2} & {\left[\left(\frac{n}{b} J_{n}\left(\alpha_{1} b\right)-\alpha_{1} J_{n+1}\left(\alpha_{1} b\right)\right) A_{1}+\left(\frac{n}{b} Y_{n}\left(\alpha_{1} b\right)-\alpha_{1} Y_{n+1}\left(\alpha_{1} b\right)\right) B_{1}+\delta J_{n+1}\left(\alpha_{2} b\right) A_{2}\right.} \\
& \left.+\delta Y_{n+1}\left(\alpha_{2} b\right) B_{2}+\frac{n}{b} J_{n}\left(\alpha_{2} b\right) A_{3}+\frac{n}{b} Y_{n}\left(\alpha_{2} b\right) B_{3}\right]-k_{r}\left(P_{n}^{s 1} H_{n}^{2^{\prime}}\left(k_{r} b\right)+P_{0} J_{n}^{\prime}\left(k_{i} b\right)\right)=0
\end{aligned}
$$


Using Eq. (A-4) from Appendix A and Eq. (27) yields:

$$
\begin{aligned}
2 \mu_{1} & {\left[A_{1}\left(\left(\frac{n(n-1)}{b^{2}}+\frac{\delta^{2}-\alpha_{1}^{2}}{2}\right) J_{n}\left(\alpha_{1} b\right)+\frac{\alpha_{1}}{b} J_{n+1}\left(\alpha_{1} b\right)\right)\right.} \\
& +B_{1}\left(\left(\frac{n(n-1)}{b^{2}}+\frac{\delta^{2}-\alpha_{1}^{2}}{2}\right) Y_{n}\left(\alpha_{1} b\right)+\frac{\alpha_{1}}{b} Y_{n+1}\left(\alpha_{1} b\right)\right) \\
& +A_{2} \delta\left(\alpha_{2} J_{n}\left(\alpha_{2} b\right)-\frac{(n+1)}{b} J_{n+1}\left(\alpha_{2} b\right)\right)+B_{2} \delta\left(\alpha_{2} Y_{n}\left(\alpha_{2} b\right)-\frac{(n+1)}{b} Y_{n+1}\left(\alpha_{2} b\right)\right) \\
& \left.+A_{3} \frac{n}{b}\left(\frac{(n-1)}{b} J_{n}\left(\alpha_{2} b\right)-\alpha_{2} J_{n+1}\left(\alpha_{2} b\right)\right)+B_{3} \frac{n}{b}\left(\frac{(n-1)}{b} Y_{n}\left(\alpha_{2} b\right)-\alpha_{2} Y_{n+1}\left(\alpha_{2} b\right)\right)\right] \\
& +P_{n}^{s 1} H_{n}^{2}\left(k_{r} b\right)+P_{0} J_{n}\left(k_{r} b\right)=0
\end{aligned}
$$

where $\mu_{1}$ is the Lame' constant of the outer layer of the cylinder. The interior acoustic field is represented by the density of $\rho_{a 2}$ and sound speed of $C_{a 2}$. The scattered sound pressure of the interior field similar to that done for the exterior field can be represented as:

$$
P_{s 2}(r, \theta, z, t)=\sum_{n=0}^{\infty} P_{n}^{s 2} \varepsilon_{n}(-i)^{n} F_{n}\left(k_{s 2} r\right) \cos (n \theta) e^{i\left(\omega t-k_{z} z\right)}
$$

where $k_{s 2}=\frac{\omega}{C_{a 2}}$ and $F_{n}$ defined as

$$
F_{n}\left(k_{s 2} r\right)=J_{n}\left(k_{s 2} r\right)+\zeta Y_{n}\left(k_{s 2} r\right)
$$

In the above representation for $F_{n}, \zeta=i$, denotes the transmitted sound pressure and $\zeta=0$ denotes the interior sound field considering the effect of interior acoustic resonance. A similar definition has been presented by Koval [16]. Using the values of $0<|\zeta|<1$, the sound absorption percentage of the interior walls can be modeled which may be scaled with the architectural absorption coefficient. This type of transmitted noise definition is used to separate the effects of structural resonances on the noise reduction from the effects of cavity resonances which will be investigated in the numerical results section. The continuity condition between internal pressure and acceleration of the structure are obtained using Eq. (A-1) in Appendix A and Eq. (30) into Eq. (26), where in Eq. (26) $P_{t}$ replaced by $P_{s 2}$, resulted as:

$$
\begin{aligned}
\rho_{a 2} \omega^{2} & {\left[\left(\frac{n}{a} J_{n}\left(\alpha_{1} a\right)-\alpha_{1} J_{n+1}\left(\alpha_{1} a\right)\right) A_{1}+\left(\frac{n}{a} Y_{n}\left(\alpha_{1} a\right)-\alpha_{1} Y_{n+1}\left(\alpha_{1} a\right)\right) B_{1}\right.} \\
& \left.+\delta J_{n+1}\left(\alpha_{2} a\right) A_{2}+\delta Y_{n+1}\left(\alpha_{2} a\right) B_{2}+\frac{n}{a} J_{n}\left(\alpha_{2} a\right) A_{3}+\frac{n}{a} Y_{n}\left(\alpha_{2} a\right) B_{3}\right]-P_{n}^{s 2} k_{s 2} J_{n}^{\prime}\left(k_{s 2} a\right)=0
\end{aligned}
$$

The relationship between the interior pressure and cylinder which is due to the acting internal pressure as a distributed force, is obtained using Eq. (A-4) from Appendix A and Eq. (30) into Eq. (27) which leads to:

$$
\begin{aligned}
2 \mu_{m} & {\left[A_{1}\left(\left(\frac{n(n-1)}{a^{2}}+\frac{\bar{\delta}^{2}-\bar{\alpha}_{2}^{2}}{2}\right) J_{n}\left(\bar{\alpha}_{1} a\right)+\frac{\bar{\alpha}_{1}}{a} J_{n+1}\left(\bar{\alpha}_{1} a\right)\right)\right.} \\
& +B_{1}\left(\left(\frac{n(n-1)}{a^{2}}+\frac{\bar{\delta}^{2}-\bar{\alpha}_{2}^{2}}{2}\right) Y_{n}\left(\bar{\alpha}_{1} a\right)+\frac{\bar{\alpha}_{1}}{a} Y_{n+1}\left(\bar{\alpha}_{1} a\right)\right) \\
& +A_{2} \bar{\delta}\left(\bar{\alpha}_{2} J_{n}\left(\bar{\alpha}_{2} a\right)-\frac{(n+1)}{a} J_{n+1}\left(\bar{\alpha}_{2} a\right)\right)+B_{2} \bar{\delta}\left(\bar{\alpha}_{2} Y_{n}\left(\bar{\alpha}_{2} a\right)-\frac{(n+1)}{a} Y_{n+1}\left(\bar{\alpha}_{2} a\right)\right) \\
& \left.+A_{3} \frac{n}{a}\left(\frac{(n-1)}{a} J_{n}\left(\bar{\alpha}_{2} a\right)-\bar{\alpha}_{2} J_{n+1}\left(\bar{\alpha}_{2} a\right)\right)+B_{3} \frac{n}{a}\left(\frac{(n-1)}{a} Y_{n}\left(\bar{\alpha}_{2} a\right)-\bar{\alpha}_{2} Y_{n+1}\left(\bar{\alpha}_{2} a\right)\right)\right] \\
& +P_{n}^{s 2} F_{n}\left(k_{s 2} a\right)=0
\end{aligned}
$$


where $\mu_{m}$ is the Lame' constant for the inner layer of a multi-layer cylinder. To calculate the transmission of the incident plane wave into a multi-layered cylinder a system of Eqs (14), (15) and (16), in which the radial boundary conditions at the inner and outer radii are changed to those presented in Eqs (28), (29), (32) and (33), are formed with the matrix dimension of $(6 \mathrm{~m}+2) \times(6 \mathrm{~m}+2)$. These equations are linear in the constants $A_{1}, A_{2}, A_{3}, B_{1}, B_{2}$, $B_{3}, P_{n}^{s 1}$ and $P_{n}^{s 2}$.

\subsection{Effect of external moving medium on the oblique incident wave}

The noise reduction results can depend on the velocity of the external moving medium. In this section the effect of external moving medium on the wave numbers of incident wave is investigated. To evaluate the effect of external moving medium on the sound transmission, the wave equation can be derived in the following form:

$$
\frac{D^{2}}{D t^{2}} P-C_{a 1}^{2} \nabla^{2} P=0
$$

where $\nabla^{2}$ is the Laplacian operator and $\frac{D}{D t}$ denotes substantial derivative time defined as:

$$
\frac{D}{D t}=V_{x} \frac{\partial}{\partial x}+V_{y} \frac{\partial}{\partial y}+V_{z} \frac{\partial}{\partial z}+\frac{\partial}{\partial t}
$$

where $V_{x}, V_{y}$ and $V_{z}$ are velocity components of the external moving medium. In the case of plane wave excitation the effect of lateral waves due to the external moving medium on the noise reduction is investigated. The lateral motion of the external medium in the $x z$ plane is presented in Fig. 1. Using Eq. (18) into Eq. (34), the wave number can be obtained as:

$$
k_{1}=\left(\frac{\omega}{\left(C_{a 1}+V \cos \left(\phi_{1}-\phi_{2}\right)\right)}\right)
$$

where $\phi_{1}$ and $\phi_{2}$ are the angles of incident wave and external flow respectively and $V$ is the amplitude of the moving medium velocity as shown in Fig. 1. Considering the Eq. (36) it is seen that the external airflow changes the wave number of the propagated mechanical waves. Also it has no effect if the direction of the external flow is perpendicular to the direction of propagation of the wave, and has the maximum effect when they are in the same direction. To evaluate the pressure field in the interior field, the wave number in the inside acoustic medium can be determined from the wave number in the axial direction. It is noted that the wave numbers of the outer and inner surfaces should be the same. Therefore the relationship between the wave numbers of the interior and exterior acoustic field obtained as:

$$
k_{s 2}=k_{1}\left(\frac{\cos \left(\phi_{1}\right)}{\cos \left(\phi_{1}^{\prime}\right)}\right)
$$

where $\phi_{1}^{\prime}$ is the angle between scattered wave and axial direction. Using Eqs (36) and (37) and considering the relationship between the $k_{z}$ and $k_{s 2}$, the wave number in the $r$ direction for the scattered wave can be obtained as follow:

$$
k_{r 2}=\left(\frac{\omega}{C_{a 2}}\right)\left(1-\left(\frac{C_{a 2} \cos \left(\phi_{1}\right)}{C_{a 1}+V \cos \left(\phi_{1}-\phi_{2}\right)}\right)\right)
$$

Evaluation of the effect of exterior moving media and the angle of incident wave on the noise reduction is investigated in the numerical results section.

\subsection{Noise reduction definition}

In this work the noise reduction is calculated using the average of sound pressure square on the interior and exterior surfaces of the cylinder which is calculated as:

$$
\bar{P}^{2}=\int_{S} P^{2}(r, \theta, z, t) d s=\iint_{S} r P^{2}(r, \theta, z, t) d \theta d z
$$


Table 1

Mechanical properties of material

\begin{tabular}{lccccc}
\hline Material & $\rho_{s}\left(\mathrm{~kg} / \mathrm{m}^{3}\right)$ & $E_{0}(\mathrm{~Pa})$ & $\nu$ & $\alpha$ & $c\left(\operatorname{Pa~}^{\alpha}\right)$ \\
\hline Aluminum & 2700 & $7.1 \mathrm{e} 10$ & 0.33 & - & - \\
Rubber & 1500 & $1.3 \mathrm{e} 08$ & 0.40 & 0.49 & $1.6 \mathrm{e} 6$ \\
\hline
\end{tabular}

where $P$ is the internal or external sound pressure acting on the body surfaces and $S$ is the related surface. Then the noise reduction $(N R)$ is calculated as:

$$
N R=10 \log _{10} \frac{\bar{P}_{\text {out }}^{2}}{\bar{P}_{\text {in }}^{2}}=10 \log _{10} \frac{b \sum_{n=0}^{\infty} \varepsilon_{n}\left(P_{0} J_{n}\left(k_{r} b\right)+P_{n}^{s 1} H_{n}^{2}\left(k_{r} b\right)\right)^{2}}{a \sum_{n=0}^{\infty} \varepsilon_{n}\left(P_{n}^{s 2} F_{n}\left(k_{s 2} a\right)\right)^{2}}
$$

where $\bar{P}_{\text {out }}^{2}$ is the time and surface average of the pressure acting on the external surface of the cylinder and $\bar{P}_{\text {in }}^{2}$ is the time and surface average of the scattered pressure on the internal surface of the cylinder. The above identification of the noise reduction prediction is simple and applicable to experimental evaluation. Also with regard to the definition of internal scattered wave function of $F_{n}$, which is included the Bessel function of the second kind, it is known on the internal surface and can be integrated.

\section{Parameters study}

The effect of air inside the cylinder on the natural frequency map is examined for a cylinder made of aluminum and then the sound transmission loss of the system is investigated. To evaluate the effect of using viscoelastic material on the noise reduction, a two layered hollow cylinder made of aluminum and a hard viscoelastic rubber is considered for the analyses. The material properties are presented in Table 1 . The parameters in the frequency power law-model fitted to the existed experimental data of a rubber are nearly similar to those reported in [27]. Aluminum is modeled as a lightly damped material with constant Young's modulus loss factor of 0.005 [2]. Structural damping is used as the damping model. The acoustic medium is standard air with a density of $1.21 \mathrm{~kg} / \mathrm{m}^{3}$ and a sound speed of $343 \mathrm{~m} / \mathrm{s}$. To validate the noise reduction results obtained from the present work, a steel cylinder is considered. The ratio of the internal to the outer radii $a / b=0.95$ with the rigid walls $(\zeta=0)$, is considered. In this analysis the first four circumferential wave numbers are considered to evaluate the monostatic back scattering noise reduction of the mentioned cylinder under a plane wave excitation. The noise reduction results calculated from the model are compared with those obtained in [9] as shown in Fig. 2. Where the non-dimensional frequency is defined as:

$$
\Omega=\frac{\omega b}{C_{a}}
$$

and $C_{a}$ is related sound speed of the medium. The agreement between the results shows the validity of the calculations.

\subsection{Effect of interior acoustic field on the natural frequencies mode map}

In this section the natural frequencies of the coupled system are calculated. According to Appendix A and the boundary conditions presented in Eqs (15) and (16) a linear algebraic equation can be generated. Natural frequencies of the system can be calculated by setting the determinant of the coefficients equal to zero. To illustrate the effect of interior acoustic field on the mode map of natural frequencies, standard air is considered as the interior acoustic medium of the aluminum cylinder. For instance, the mode map for circumferential wave number of one $(n=1)$ is illustrated in Fig. 3. The results show that 5th and 6th natural frequencies of the air medium are coupled with that of the 2nd solid mode map. The mode maps for the coupled system are changed as shown in Fig. 3. For example in this case the part of the 2nd solid mode map belongs to the 7th mode map of the coupled system and the rest of that follows the 8th mode map. Then for the noise reduction evaluation of a coupled system, with regard to the mode maps, all of the resonances frequencies should be taken into account. This consideration is studied in the following section. 


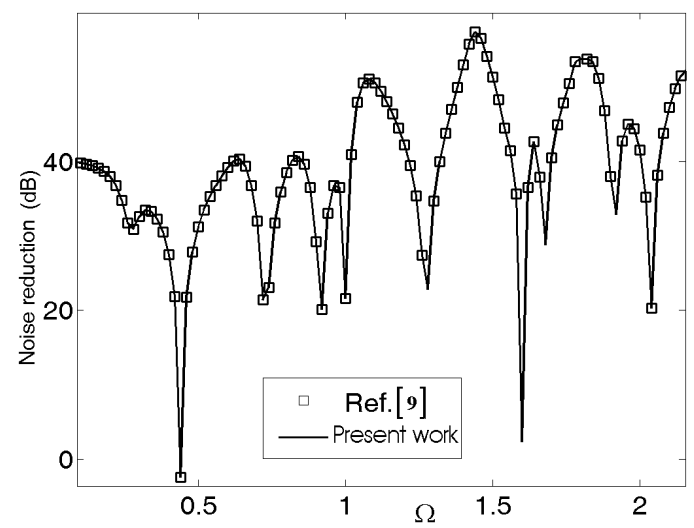

Fig. 2. Comparison of the noise reduction obtained from Ref. [9] with those obtained from the present work for the case of monostatic back scattering of the steel cylinder.

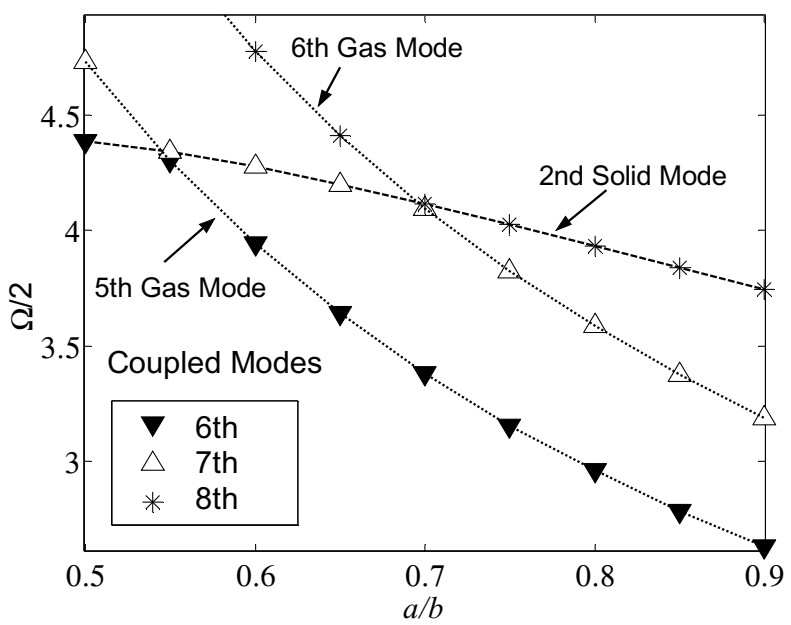

Fig. 3. Non-dimensional resonance frequency, vs geometry for circumferential wave number one, of the coupled air filled cylinder.

\subsection{Noise reduction in non external flow condition}

In this part some considerations in the noise reduction calculation of a coupled system are investigated. The effect of cavity resonance, frequency independent viscoelastic material (VEM) and moving exterior medium on the noise reduction are calculated.

\subsubsection{Noise reduction considering cavity resonances}

A hollow aluminum circular cylinder with $a / b=0.994$ exposed to the vertical incident plane wave is considered. In this case the noise reduction can be obtained using Eq. (40). Figure 3 shows that in light gas such as air, high modes of the acoustical medium are coupled with the low modes of the structure. Then to obtain the noise reduction in a frequency bandwidth, the minimum number of terms which must be used in the series to consider the effect of cavity resonances on the noise reduction, depends on the zeros of the derivative of $F_{n}\left(k_{s 2} r\right)$, with respect to the related argument, for interior acoustic resonances with the rigid walls $(\zeta=0)$. Figure 4-b shows the amplitude of the derivative of the function $F_{n}$ verses non-dimensional frequency. The zeros of the function $F_{n}$ can be recognized to represent the resonance frequencies of the cavity. Figure 4-b shows that in the frequency range the circumferential wave number $n \geqslant 6$, has no resonances of the interior acoustic field. Therefore, the minimum terms that capture the 


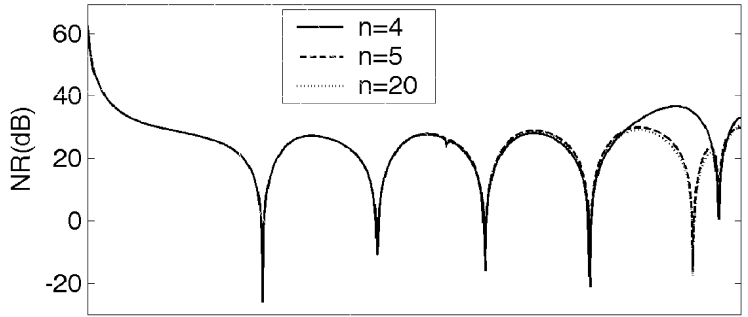

(a)

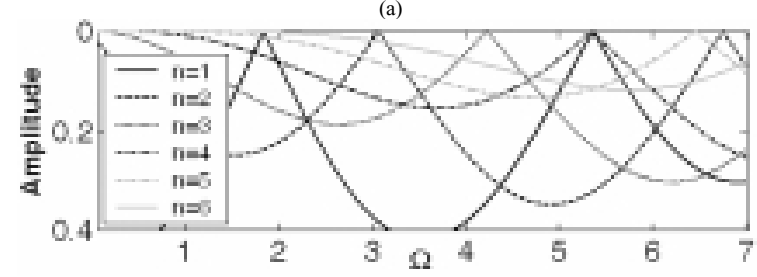

(b)

Fig. 4. The effect of cavity resonances on noise reduction for a hollow aluminum cylinder with $a / b=0.994$, (a) The noise reduction for three values of circumferential wave number (b) The amplitude of the $F_{n}^{\prime}$ vs non-dimensional frequency.

Table 2

Mechanical properties of the rubber in three frequencies

\begin{tabular}{ccc}
\hline Frequency $(\mathrm{Hz})$ & $E_{d}(\mathrm{MPa})$ & $\eta$ \\
\hline 10 & 152.6 & 0.14 \\
1000 & 345.9 & 0.61 \\
10000 & 797.0 & 0.81 \\
\hline
\end{tabular}

entire cavity resonances are the first five wave numbers. Also by considering insufficient number of modes in the calculations as shown for $n=4$ in Fig. 4-a, the achieved noise reduction is greater than the accurate value (between non-dimensional frequencies 6 and 7), such as that obtained in [9]. In this reference only the first three acoustical modes were considered. In addition, due to the coupling of the higher modes of the acoustic medium with the lower modes of the structure, as shown in Fig. 3, the internal acoustic resonances are more important than those of the structural modes in the interested frequency range for noise reduction analyses.

\subsubsection{Constant and frequency dependent VEM}

The loss factor of lightly damped materials such as metals can be considered constant with no noticeable error in results [27]. The property of viscoelastic materials can be assumed constant in a narrow band frequency but not over a wide frequency range. In this part a viscoelastic single layer cylinder with the boundary conditions explained in Section 4-2 is considered. To evaluate the effect of constant and frequency dependent VEM properties on the noise reduction, the hard rubber presented in Table 1 is used for the VEM cylinder with $a / b=0.994$ exposed to the vertical plane wave. The related viscoelastic material properties are presented in Fig. 5. Three loss factors considered for the noise reduction calculation are in Table 2, obtained from the power law method defined by Eq. (3). It should be noted that in the current and following parts the effect of cavity resonance on the noise reduction has been disregarded in the calculations. Therefore the interior medium of the cylinder has been considered to be perfectly absorbent $(\zeta=i)$. This consideration provides the capability to study the effects of different parameters on the noise reduction individually. The noise reduction without the cavity resonance effect is calculated and depicted in Fig. 6. This figure shows that considering constant viscoelastic material properties causes noticeable differences at low frequencies and near to the resonance frequency of the structure. But in the rest of the frequency range (higher than 6), the difference becomes small. Therefore, the constant value can be used for the viscoelastic properties only in the high frequency range far from the structural resonances zone. 


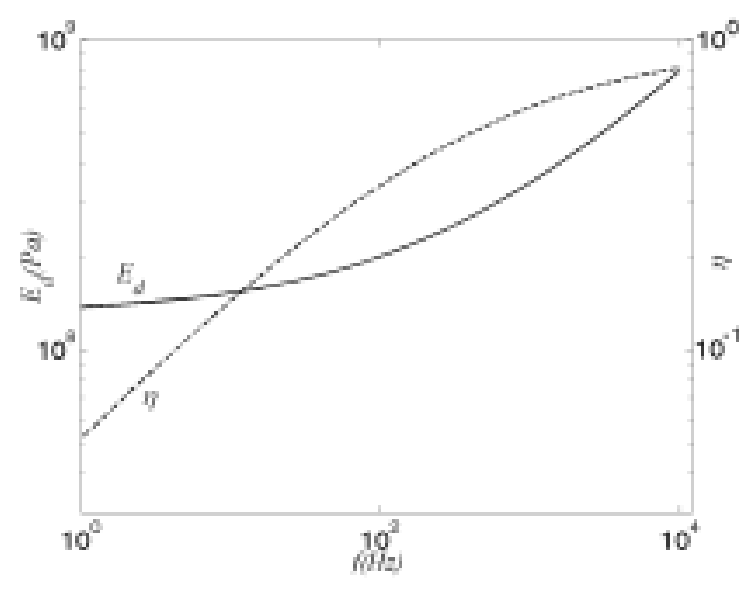

Fig. 5. Frequency dependent properties of the viscoelastic material.

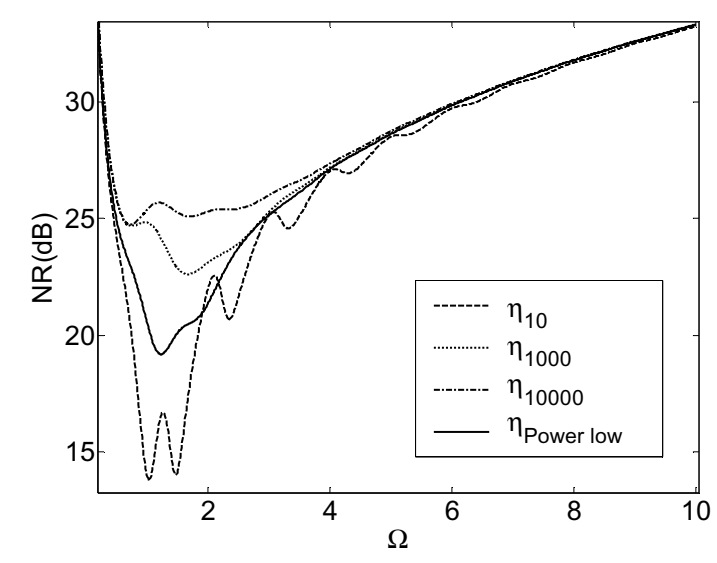

Fig. 6. Comparison between the noise reductions obtained from the constant and frequency dependent viscoelastic materials.

\subsubsection{Comparison of the VEM and aluminum layers}

To evaluate the effect of the internal viscoelastic layer on the noise reduction, two-layered hollow cylinders are considered. The external layer is made of aluminum for all models. But, the internal layer is made of aluminum or viscoelastic material in various models. The boundary conditions on the internal and external faces of the models are the same as those presented in the previous section. The boundary conditions presented in Eq. (14) are also applied between the layers of the cylinders. In these models, the thickness of the external cylinder remains unchanged. The thickness of the internal layer made of viscoelastic material (VEM) is selected based on the internal layer thickness of the two-layered aluminum model with the condition that the total weight of the body remains constant comparing with the fully aluminum models. Therefore, the relation between the thicknesses of the two different materials with equal weight in cylindrical shape is defined as:

$$
\Delta_{V E M}=a-\left(a^{2}-\frac{\rho_{A l} \Delta_{A l}\left(2 a-\Delta_{A l}\right)}{\rho_{V E M}}\right)^{1 / 2}
$$

where $\Delta$ is the thickness of the layer. In these analyses three models with various external and internal layers thicknesses are considered.

In the first model, an aluminum cylinder with the external layer thickness of $3 \mathrm{~mm}$ and $a / b=0.994$ with an aluminum internal layer of $1 \mathrm{~mm}$ thickness is considered. The obtained noise reduction due to the use of $1 \mathrm{~mm}$ internal aluminum layer is compared with those obtained using internal viscoelastic material layers with the thicknesses of 


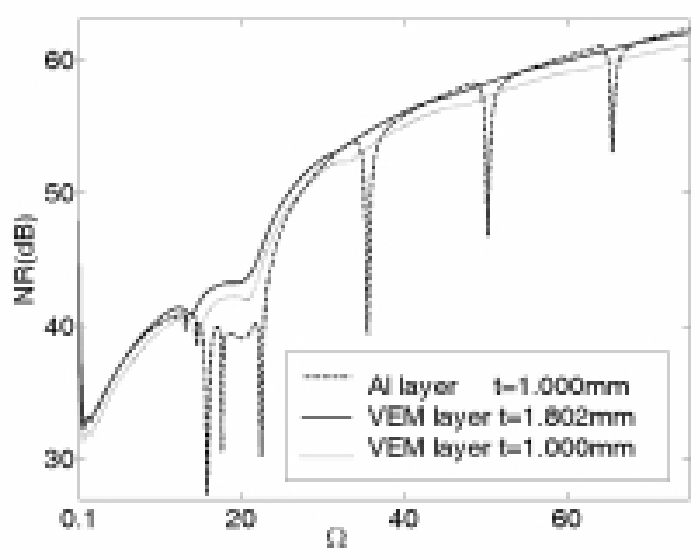

Fig. 7. Noise reduction of the aluminum cylinders with the thickness of $3 \mathrm{~mm}$ and $a / b=0.994$ with various internal layers.

$1.0 \mathrm{~mm}$ and $1.802 \mathrm{~mm}$. It is noted that the weight of viscoelastic layer with the thickness of $1.802 \mathrm{~mm}$ is equal to the aluminum internal layer of $1.0 \mathrm{~mm}$ thickness. Figure 7 shows that the noise reduction obtained using both VEM internal layers are almost similar to those obtained from that using the aluminum internal layer at very low frequencies. But at the middle range of the presented frequencies the noise reduction obtained using the VEM layers is better than that obtained from the aluminum layer by the magnitudes of about 1.5 to $3 \mathrm{~dB}$ in the non-dimensional frequency of 8-30. In general Fig. 7 shows that the VEM is more effective than the aluminum layer in reducing the effect of structural resonances on the noise reduction. At high frequencies, the effect of using the VEM internal layer for noise reduction is less than that using the aluminum internal layer with the same thickness, but it is useful for decreasing the effects of structural resonances on the obtained noise reduction.

A second model, a thicker cylinder with the thickness of $50 \mathrm{~mm}$ and $a / b=0.9$, is considered. An aluminum layer of $10 \mathrm{~mm}$ and viscoelastic internal layers of $10 \mathrm{~mm}$ and $18.17 \mathrm{~mm}$ thicknesses are considered as three individual internal layers. The VEM layer of $18.17 \mathrm{~mm}$ thickness has the same weight as the $10 \mathrm{~mm}$ thickness aluminum layer. The results presented in Fig. 8 show that the noise reduction obtained using aluminum internal layer is slightly more than those obtained using VEM internal layer at very low frequencies. But as the frequency increases the results obtained using the VEM internal layer are better. Furthermore using the viscoelastic internal layer is successful in reducing the effect of structural resonances on the noise reduction as shown in Fig. 8. Comparing the results presented in Fig. 8 and those in Fig. 7 shows that using a VEM internal layer with the same thickness of an aluminum internal layer, a better noise reduction in higher frequencies is obtained for thick cylinders in comparison with those obtained for thinner cylinder (Fig. 7).

A third investigation a thick cylinder with the thickness of $100 \mathrm{~mm}$ and $a / b=0.8$ internally covered by an aluminum layer of $50 \mathrm{~mm}$, the VEM layers of $50 \mathrm{~mm}$ and $95.86 \mathrm{~mm}$ thicknesses, is considered. The internal viscoelastic layer of $95.86 \mathrm{~mm}$ thickness has the same weight as the aluminum internal layer. The noise reduction results of the cylinders with these internal layers are presented in Fig. 9. This figure shows that the noise reduction results have the same trend as those obtained for the previous thick cylinder in Fig. 8. This figure also shows that at very low frequencies using the aluminum internal layer is more effective than using VEM internal layer for noise reduction. But by increasing the frequency, the noise reduction obtained using VEM internal layer is higher than that obtained from using an aluminum internal layer. The effect of the structural resonances on the noise reduction is the same as the before mentioned cases. The noise reductions obtained using the VEM and aluminum internal layers with the same thicknesses become almost the same at high frequencies.

Based on the investigated cases in this section, it can be concluded that using the viscoelastic internal layer instead of the aluminum layer with the equal weight results in a better noise reduction in thin cylinders. A better noise reduction at high frequencies can be obtained when a viscoelastic layer is used instead of a metal internal layer with the same thickness. 


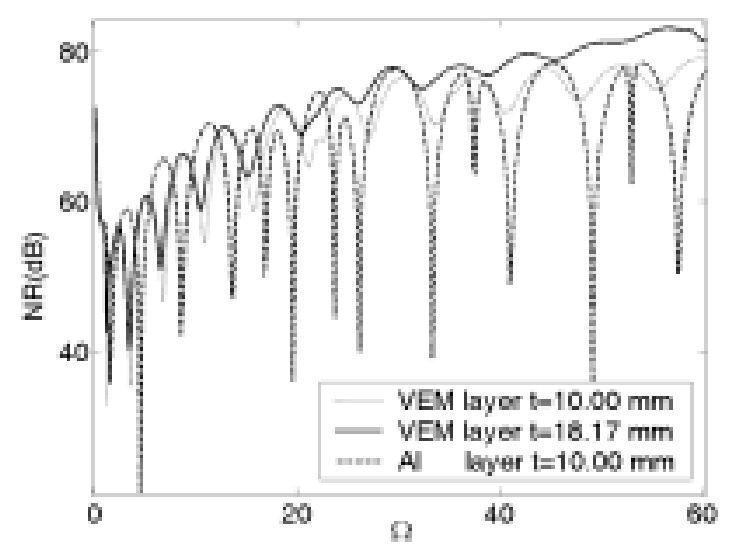

Fig. 8. Noise reduction for an aluminum cylinder with the thickness of $50 \mathrm{~mm}$ and $a / b=0.9$ with various internal layers.

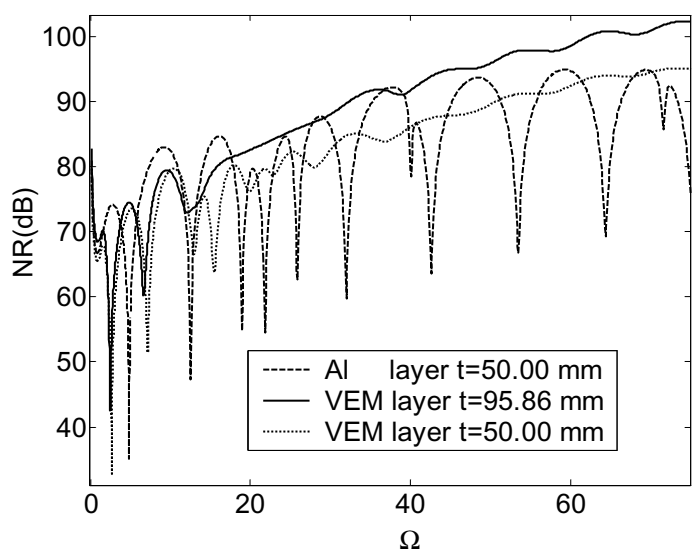

Fig. 9. Noise reduction for an aluminum cylinder with the thickness of $100 \mathrm{~mm}$ and $a / b=0.8$ with various internal layers.

\subsubsection{Effect of VEM internal layer thickness on noise reduction}

In this section, the effect of the ratio between the thickness of the VEM internal layer and the structural base thickness on the noise reduction is studied for the aluminum cylinder with the thickness of $3 \mathrm{~mm}$ and $a / b=0.994$. Differences of the noise reduction $(d N R)$ calculated from the following equation, Eq. (43), are studied as a function of the ratio of the VEM internal layer to the structural thickness.

$$
d N R=\overline{N R}_{i}-\overline{N R}_{0}
$$

where $\overline{N R}_{i}$ is the average of the noise reduction related to the use of VEM internal layer and $\overline{N R}_{0}$ is the average of the noise reduction in the aluminum structure without using the viscoelastic internal layer. The results are shown in Fig. 10, and are presented in two non-dimensional frequency bands: below 10, and between 10 and 60 . It can be seen that the noise reduction increases nonlinearly with increasing thickness of the viscoelastic internal layer. At low frequencies below 10, the increase of the average of the noise reduction is smaller than that obtained for the high frequencies of more than 10. It can also be seen that around the thickness ratio of 2.5 and bigger, increasing the VEM thickness is not useful for noise reduction purposes. Finally, it is concluded that using VEM internal layers is less effective in the non-dimensional frequency range lower than 10 for the range between 10 and 60 .

\subsubsection{Using VEM as an internal or external layer}

Using the VEM layer as an internal or external layer for a base structure of a hollow cylinder is usually dictated by the type of application and existed constrained in the structure. The effect of using the viscoelastic layer as the 


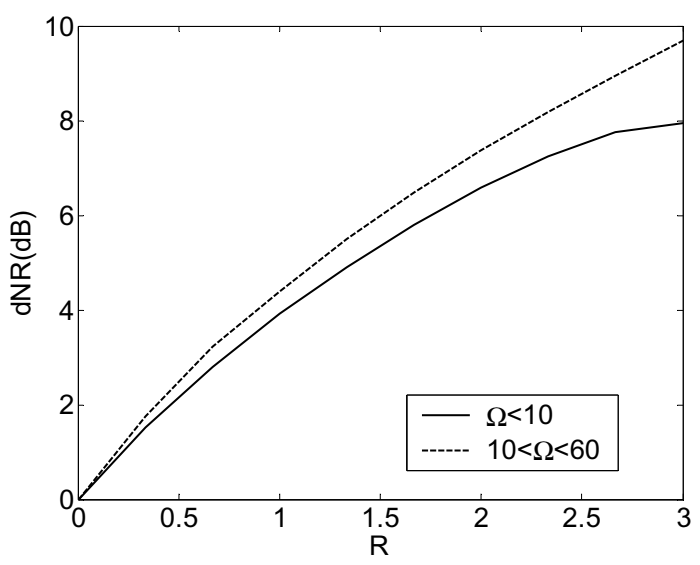

Fig. 10. Noise reduction versus the ratio of VEM layer to the base cylinder thicknesses (R) for an aluminum cylinder with the thickness of $3 \mathrm{~mm}$ and $a / b=0.994$.

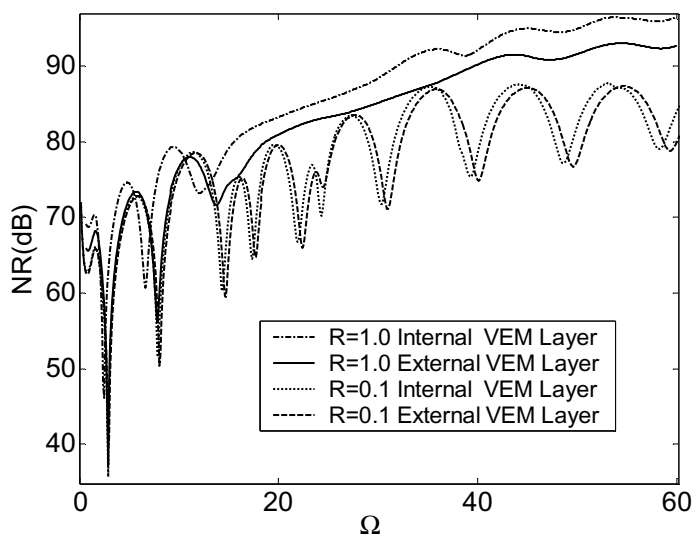

Fig. 11. Noise reduction obtained for the cylinder with the thicknesses of $10 \mathrm{~mm}$ using the VEM as an internal or external layer.

internal or external layer on noise reduction is examined for thin and thick VEM layers. The noise reductions are presented for four different cases in Fig. 11, where R is the ratio of VEM layer thickness to the aluminum cylinder thickness. In these analyses the aluminum cylinder thickness of $10 \mathrm{~mm}$ and $a / b=0.8$ is considered. The results obtained using the thin VEM layers $(R=0.1)$ in this figure shows that the differences between the obtained noise reductions using the VEM as an internal layer or an external layer are not noticeable. In the case of using thick layers $(R=1.0)$, this figure shows that using the VEM layer on the internal surface of the structure is more effective than using it on the external surface of the cylinder at almost all frequencies. It is noted that the noise reductions using the external layer with $R=1.0$ at the frequencies lower than 10 are almost similar to those obtained using $R=0.1$. In the non-dimensional frequency range of between 10 and 60 for $R=1.0$, using the external VEM layer causes the average of obtained noise reduction about $4 \mathrm{~dB}$ less than that using the internal layer with the same thicknesses. It can be explained that this phenomenon is attributable to the reduction of the internal radius of the cylinder when the VEM layer is used. The geometrical changes of the cylinder such as internal radius, affect the acoustic impedance of the interior medium.

\subsection{Effect of exterior moving medium on noise reduction}

To evaluate effect of the incident angle of the wave and external flow on the sound transmission, the aforementioned two layered cylinder made of aluminum base cylinder with the thickness of $3 \mathrm{~mm}, a / b=0.994$ and the VEM internal 


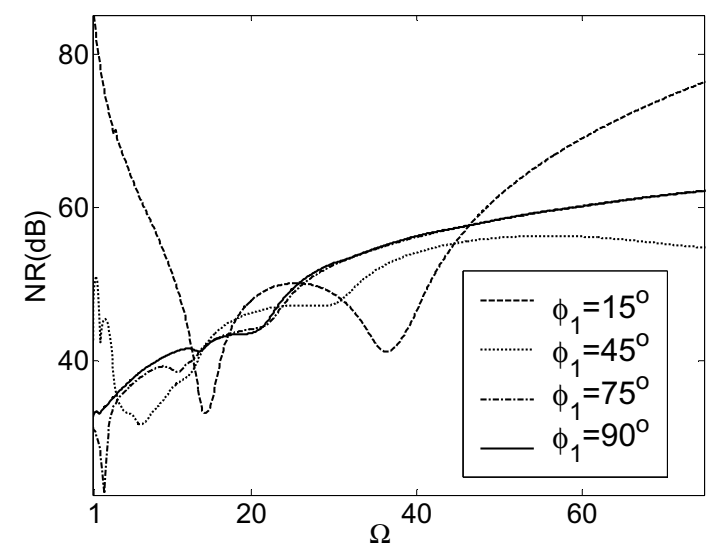

Fig. 12. Effects of incident angle of plane wave on the noise reduction for a two layered cylinder.

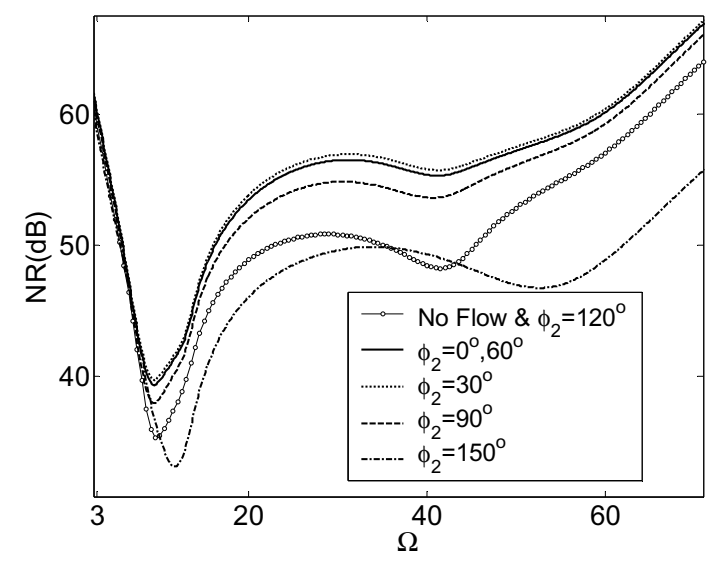

Fig. 13. Effects of the incident angle of the exterior airflow on noise reduction.

layer with the thickness of $2 \mathrm{~mm}$ is considered. The effect of the various incident angle of the plane wave on the noise reduction of this two layered cylinder is obtained and depicted in Fig. 12. This figure shows that the noise reduction trends for the angle of incidents of below $45^{\circ}$ are almost the same and those obtained for the incident angle of higher than $45^{\circ}$. At the very low and high frequency ranges, by decreasing in the angle of incident below $45^{\circ}$, the noise reduction increases extremely. It should be noted that the results for the angles of greater than $90^{\circ}$ are repeated symmetrically with respect to the $x$ coordinate as shown in Fig. 1.

To evaluate the effect of exterior medium motion on the noise reduction a unit plane wave incident to a two layered cylinder with the aforementioned geometry $\left(\phi_{1}=30^{\circ}\right)$ is considered. The results for various angles of incident airflow with the velocity of 0.2 times of the sound speed are presented in Fig. 13. This figure shows that the maximum differences of the noise reductions between the conditions of no flow and external airflow occurs around the structural frequencies resonances and it occurs at airflow angle of $30^{\circ}$ which is equal to the incident angle of considered plane wave. The noise reduction for different angles of incident medium becomes nearly constant, 3 $\mathrm{dB}$, when the frequency increases beyond 30. But at the angle of $150^{\circ}$, the noise reduction decreases about $5 \mathrm{~dB}$ with respect to the no flow condition. It becomes a nearly constant value of $8 \mathrm{~dB}$ at higher frequencies around the structural resonance frequency.

To evaluate the effect of exterior velocity value on the noise reduction, the cylinder with $\phi_{\mathrm{x}}=30^{\circ}$ and $\phi_{\mathrm{y}}=0^{\circ}$ is considered for different values of the flow velocities. The results are presented in Fig. 14. This figure shows that the noise reduction increases with the external flow velocity. The noise reduction increases significantly for the flow velocity of less than 0.4 times of the sound speed, and the effect of the external flow on the noise reduction decreases for the flow velocities of higher than 0.4 . 


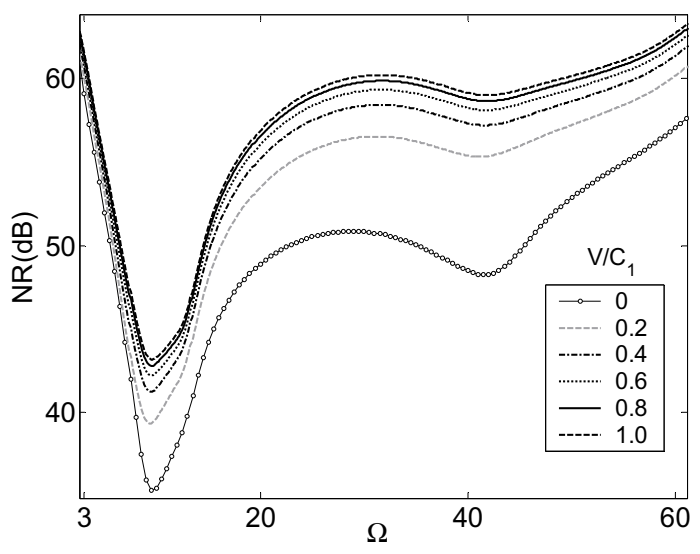

Fig. 14. Effects of different external medium velocity on the noise reduction.

\subsection{Practical consideration in evaluation of noise reduction}

In this section, the calculation procedure for the noise reduction analyses of an infinite cylinder is modified to analyze two practical cases. To evaluate the results obtained from the theoretical method some consideration may be useful to match the situation of cylinders found in practice. Two considerations of anechoic internal medium and the plane wave incident on an infinite cylinder are presented in this section.

\subsubsection{Interior absorption}

Two types of noise reduction analyses may be performed for various problems. The first one is considering the internal wall of a cylinder which is acoustically rigid and the second one is considering an anechoic condition for the interior medium. Using Eq. (31) with the values of $\zeta$ equal to 0 and $i$ provides these two conditions respectively, although the unit value of $\zeta$ is useful to evaluate the effect of the cylinder structure on noise reduction and to avoid considering of the interior acoustic cavity resonances on the results. But in practice making a fully anechoic condition in a cylinder is too difficult and these results may not be obtained as a result of practical condition such as those presented in [7]. By choosing a value of $0<|\zeta|<1$, it is possible to scale the results with the real condition of interior sound absorption such as architecture absorption coefficient. The noise reductions obtained for different values of $\zeta$ are presented in Fig. 15. The geometry and properties of the two-layered cylinder are the same as those presented in Section 5-3. This figure shows that by choosing a value of $\zeta$ the percentage effect of the interior acoustic cavity resonances on the noise reduction can be determined. It is important to note that this type of absorption is different from considering fluid absorption which obtained by choosing a complex value for density of medium and can be scaled with the structural damping.

\subsubsection{Noise reduction of a periodically uniform incident wave}

The three-dimensional elasticity solution for the infinite cylinder is provided to evaluate the vibration of cylinders under excitations which periodically applied in the axial direction of the cylinder. Another application of this solution is the modeling of a cylinder as a part of the infinite cylinder. In the axial direction the exponential function can be separated into two solution forms of symmetric and antisymmetric as mentioned before. Considering the first form solution for $T(\delta z)$, cosine function, a boundary condition obtained by constraining the displacement in the axial direction at the ends $\left(u_{z}=0\right)$. In the practical situation, applying the supports on a cylinder covers a part of the cylinder which masks this part from the incident wave as shown in Fig. 16. To evaluate the effect of this condition on the noise reduction results, a two-layered cylinder with the half-length to outer-radius ratio $(l / b)$ of one is considered. The external and internal layers are made of aluminum and VEM respectively. The geometry and properties of the cylinder are the same as those presented in previous section. The Fourier series of a uniform wave which is distributed from $-z_{0}$ to $z_{0}$ in the $z$ direction can be expressed as: 


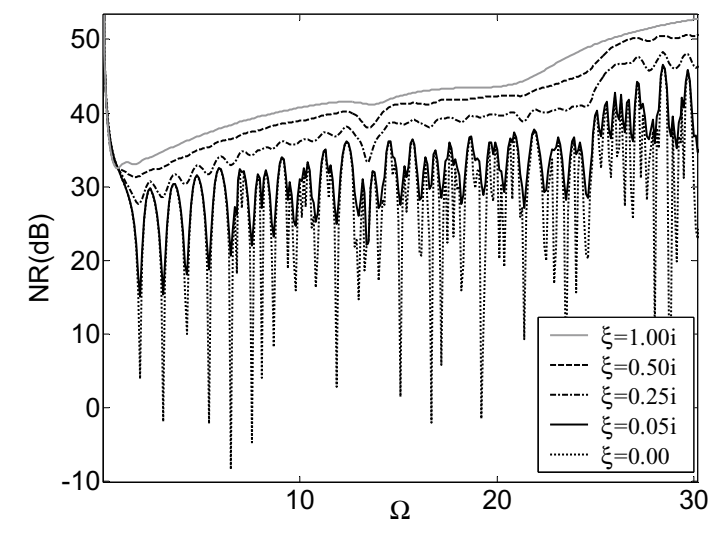

Fig. 15. Noise reduction for different absorption coefficient.

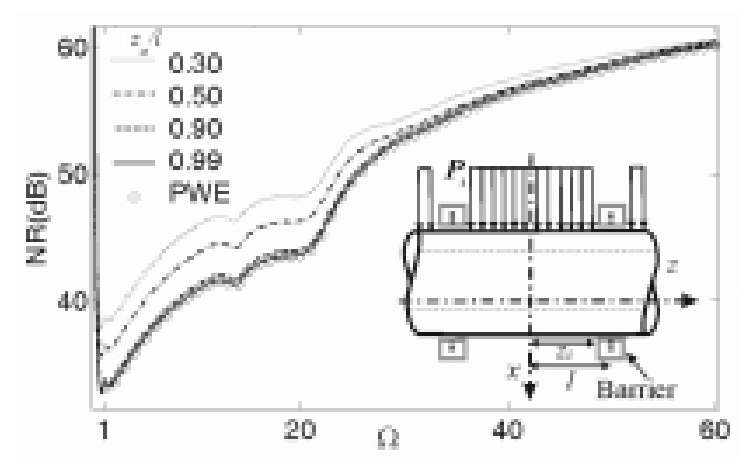

Fig. 16. Noise reduction of infinite cylinder with $l / b=1$ under uniform wave excitation with different $\not / l$, PWE demonstrates plane wave excitation.

$$
P_{i}(r, \theta, z)=P_{0}\left(\frac{z_{0}}{l}+\sum_{k=1}^{\infty} \frac{2}{k \pi} \sin \left(\frac{k \pi z_{0}}{l}\right) \cos \left(\frac{k \pi z}{l}\right)\right) \sum_{n=0}^{\infty} \varepsilon_{n}(-i)^{n} J_{n}\left(k_{r} r\right) \cos (n \theta)
$$

where $k$ is the axial wave number, $l$ is half length of the cylinder and $z_{0}$ is the percentage of the half-length exposed to the incident wave. In this case, the sound scattering equations presented in Eqs (25) and (30) are changed to the following equations:

$$
\begin{aligned}
& P_{s 1}(r, \theta, z, t)=\sum_{n=0}^{\infty} \sum_{k=0}^{\infty} P_{n k}^{s 1} \varepsilon_{n}(-i)^{n} H_{n}^{2}\left(k_{r} r\right) \cos \left(\frac{k \pi z}{l}\right) \cos (n \theta) e^{i \omega t} \\
& P_{s 2}(r, \theta, z, t)=\sum_{n=0}^{\infty} \sum_{k=0}^{\infty} P_{n k}^{s 2} \varepsilon_{n}(-i)^{n} F_{n}\left(k_{s 2} r\right) \cos \left(\frac{k \pi z}{l}\right) \cos (n \theta) e^{i \omega t}
\end{aligned}
$$

Therefore, the definition of the noise reduction can be expressed as:

$$
N R=10 \log _{10} \frac{b \sum_{n=0}^{\infty} \sum_{k=0}^{\infty} \varepsilon_{n} \varepsilon_{k}\left(\frac{2 P_{0}}{k \pi} J_{n}\left(k_{r} b\right) \sin \left(\frac{k \pi z_{0}}{l}\right)+P_{n k}^{s 1} H_{n}^{2}\left(k_{r} b\right)\right)^{2}}{a \sum_{n=0}^{\infty} \sum_{k=0}^{\infty} \varepsilon_{n} \varepsilon_{k}\left(P_{n k}^{s 2} F_{n}\left(k_{s 2} a\right)\right)^{2}}
$$

where $\varepsilon_{k}$ are defined as follows:

$$
\begin{array}{ll}
\varepsilon_{k}=2 & \text { for } k=0 \\
\varepsilon_{k}=1 & \text { for } k \geqslant 1
\end{array}
$$


The noise reductions for various values of $z_{0} / l$ ratio with $l / b=1$ and $\zeta=i$ are presented in Fig. 16. This figure shows that by increasing the $z_{0} / l$ ratio the noise reductions are similar to those obtained from the plane wave analysis of an infinite cylinder. To prevent clutter of the graphs, noise reductions obtained for the $z_{0} / l$ ratios of between $0.9-0.99$ are not presented here. In the non-dimensional frequency range of less than 30 , when $z_{0} / l>0.9$, the differences between the noise reduction obtained for the infinite cylinder under the plane wave excitation and those presented in Fig. 16 are less than $1 \mathrm{~dB}$ and when the ratio of $z_{0} / l$ is greater than 0.95 are less than $0.5 \mathrm{db}$. Figure 16 shows that the noise reduction results obtained for the case with $z_{0} / l=0.99$ is in good agreement with that obtained from a plane wave excitation of the infinite cylinder, the differences between the obtained results from the two solutions are less than $0.02 \mathrm{db}$. For the non-dimensional frequency range of more than 30, the differences tend to decrease asymptotically to become constant. It can be seen that the noise reduction differences between the plane wave and uniform periodical wave is less than $1 \mathrm{~dB}$ for the case of $z_{0} / l=0.9$. This value decreases as $l / b$ increases, e.g.: for $l / b=10$, the differences reduce to less than $0.2 \mathrm{~dB}$. Choosing the holders in a form that covered less than 0.1 times the cylinder length, the results should be close to those obtained for the ideal form of the plane wave excitation.

\section{Conclusion}

An analytical solution as well as numerical results concerning the interaction of a plane wave with a viscoelastic infinite cylinder coupled with internal and external air media was presented. The noise reduction was calculated in the context of sound transmission into an infinite cylinder subjected to the incident plane wave and external airflow. It was shown that considering properties of the viscoelastic material to be frequency independent causes some errors in the noise reduction calculation in the low frequency range and around the structural resonance. The results also showed that using a viscoelastic material with high loss factor leads to a uniform noise reduction and the effects of structural resonances is reduced, especially in the middle and high frequency ranges. Using viscoelastic material on the internal or external surface of an aluminum cylinder is important for thick cylinders. The study of the effects of direction and the value of external airflow velocity showed that the noise reduction obtained in the non-flow condition should be modified for practical conditions which may cause some differences in the results. Using the $\zeta$ coefficient for noise reduction calculation is a useful parameter to control the percentage of the internal acoustic field resonances effect on the noise reduction. The evaluation of noise reduction of the infinite cylinder which is partially masked from the uniform incident wave provides some insights in modeling the practical systems subjected to the incident plane wave.

\section{Appendix A}

The displacement and stress components can be expressed in complex form in $z$ direction as:

$$
\begin{aligned}
u_{r}= & \left\{\left(\frac{n}{r} J_{n}\left(\alpha_{1} r\right)-\alpha_{1} J_{n+1}\left(\alpha_{1} r\right)\right) A_{1}+\left(\frac{n}{r} Y_{n}\left(\alpha_{1} r\right)-\alpha_{1} Y_{n+1}\left(\alpha_{1} r\right)\right)\right. \\
& B_{1}+\delta J_{n+1}\left(\alpha_{2} r\right) A_{2}+\delta Y_{n+1}\left(\alpha_{2} r\right) B_{2} \\
& \left.+\frac{n J_{n}\left(\alpha_{2} r\right)}{r} A_{3}+\frac{n Y_{n}\left(\alpha_{2} r\right)}{r} B_{3}\right\}\left\{\begin{array}{l}
\cos (n \theta) \\
\sin (n \theta)
\end{array}\right\} e^{i(\omega t-\delta z)} \\
u_{\theta}= & \left\{-\frac{n J_{n}\left(\alpha_{1} r\right)}{r} A_{1}-\frac{n Y_{n}\left(\alpha_{1} r\right)}{r} B_{1}+\delta J_{n+1}\left(\alpha_{2} r\right) A_{2}+\delta Y_{n+1}\left(\alpha_{2} r\right)\right. \\
& B_{2}-\left(\frac{n}{r} J_{n}\left(\alpha_{2} r\right)-\alpha_{2} J_{n+1}\left(\alpha_{2} r\right)\right) A_{3} \\
& \left.-\left(\frac{n}{r} Y_{n}\left(\alpha_{2} r\right)-\alpha_{2} Y_{n+1}\left(\alpha_{2} r\right)\right) B_{3}\right\}\left\{\begin{array}{c}
\sin (n \theta) \\
\cos (n \theta)
\end{array}\right\} e^{i(\omega t+\delta z)}
\end{aligned}
$$




$$
\begin{aligned}
& u_{z}=-\left\{\delta J_{n}\left(\alpha_{1} r\right) A_{1}+\delta Y_{n}\left(\alpha_{1} r\right) B_{1}+\alpha_{2} J_{n}\left(\alpha_{2} r\right) A_{2}+\alpha_{2} Y_{n}\left(\alpha_{2} r\right) B_{2}\right\}\left\{\begin{array}{l}
\cos (n \theta) \\
\sin (n \theta)
\end{array}\right\} e^{i(\omega t-\delta z)} \\
& \sigma_{r r}=2 \mu\left[A_{1}\left(\left(\frac{n(n-1)}{r^{2}}+\frac{\delta^{2}-\alpha_{1}^{2}}{2}\right) J_{n}\left(\alpha_{1} r\right)+\frac{\alpha_{1}}{r} J_{n+1}\left(\alpha_{1} r\right)\right)\right. \\
& +B_{1}\left(\left(\frac{n(n-1)}{r^{2}}+\frac{\delta^{2}-\alpha_{1}^{2}}{2}\right) Y_{n}\left(\alpha_{1} r\right)+\frac{\alpha_{1}}{r} Y_{n+1}\left(\alpha_{1} r\right)\right) \\
& +A_{2} \delta\left(\alpha_{2} J_{n}\left(\alpha_{2} r\right)-\frac{(n+1)}{r} J_{n+1}\left(\alpha_{2} r\right)\right)+B_{2} \delta\left(\alpha_{2} Y_{n}\left(\alpha_{2} r\right)-\frac{(n+1)}{r} Y_{n+1}\left(\alpha_{2} r\right)\right) \\
& \left.+A_{3} \frac{n}{r}\left(\frac{(n-1)}{r} J_{n}\left(\alpha_{2} r\right)-\alpha_{2} J_{n+1}\left(\alpha_{2} r\right)\right)+B_{3} \frac{n}{r}\left(\frac{(n-1)}{r} Y_{n}\left(\alpha_{2} r\right)-\alpha_{2} Y_{n+1}\left(\alpha_{2} r\right)\right)\right] \\
& \left\{\begin{array}{l}
\cos (n \theta) \\
\sin (n \theta)
\end{array}\right\} e^{i(\omega t-\delta z)} \\
& \sigma_{r \theta}=\mu\left[A_{1} \frac{2 n}{r}\left(\frac{(1-n)}{r} J_{n}\left(\alpha_{1} r\right)+\alpha_{1} J_{n+1}\left(\alpha_{1} r\right)\right)+B_{1} \frac{2 n}{r}\left(\frac{(1-n)}{r} Y_{n}\left(\alpha_{1} r\right)+\alpha_{1} Y_{n+1}\left(\alpha_{1} r\right)\right)\right. \\
& +A_{2} \delta\left(\alpha_{2} J_{n}\left(\alpha_{2} r\right)-\frac{2(n+1)}{r} J_{n+1}\left(\alpha_{2} r\right)\right)+B_{2} \delta\left(\alpha_{2} Y_{n}\left(\alpha_{2} r\right)-\frac{2(n+1)}{r} Y_{n+1}\left(\alpha_{2} r\right)\right) \\
& +A_{3}\left(\left(\alpha_{2}^{2}-\frac{2 n(n-1)}{r^{2}}\right) J_{n}\left(\alpha_{2} r\right)-\frac{2 \alpha_{2}}{r} J_{n+1}\left(\alpha_{2} r\right)\right) \\
& \left.+B_{3}\left(\left(\alpha_{2}^{2}-\frac{2 n(n-1)}{r^{2}}\right) Y_{n}\left(\alpha_{2} r\right)-\frac{2 \alpha_{2}}{r} Y_{n+1}\left(\alpha_{2} r\right)\right)\right]\left\{\begin{array}{l}
\sin (n \theta) \\
\cos (n \theta)
\end{array}\right\} e^{i(\omega t+\delta z)} \\
& \sigma_{r z}=\mu\left[2 A_{1} \delta\left(-\frac{n}{r} J_{n}\left(\alpha_{1} r\right)+\alpha_{1} J_{n+1}\left(\alpha_{1} r\right)\right)+2 B_{1} \delta\left(\frac{n}{r} Y_{n}\left(\alpha_{1} r\right)-\alpha_{1} Y_{n+1}\left(\alpha_{1} r\right)\right)\right. \\
& +A_{2}\left(-\frac{n \alpha_{2}}{r} J_{n}\left(\alpha_{2} r\right)+\left(\alpha_{2}^{2}-\delta^{2}\right) J_{n+1}\left(\alpha_{2} r\right)\right)+B_{2}\left(-\frac{n \alpha_{2}}{r} Y_{n}\left(\alpha_{2} r\right)+\left(\alpha_{2}^{2}-\delta^{2}\right) Y_{n+1}\left(\alpha_{2} r\right)\right) \\
& \left.-\frac{n \delta}{r}\left(A_{3} J_{n}\left(\alpha_{2} r\right)+B_{3} Y_{n}\left(\alpha_{2} r\right)\right)\right]\left\{\begin{array}{c}
\cos (n \theta) \\
\sin (n \theta)
\end{array}\right\} e^{i(\omega t+\delta z)}
\end{aligned}
$$

\section{References}

[1] A.E. Armenakas, Propagation of harmonic waves in composite circular cylindrical shells, I: Theoretical investigations, AIAA Journal 5 (1967), 740-744.

[2] C. Niezrecki, Structural \& Internal Acoustic Response of Cylinders with Applications to Rocket Payload Fairings, PhD thesis, Virginia Polytechnic, 1999

[3] D.A. Bofilios and C.S. Lyrintzis, Structure-borne noise transmission into cylindrical enclosures of finite extent, AIAA Journal 29(8) (1991), 1193-1201.

[4] D. Rao Mohan, Recent applications of viscoelastic damping for noise control in automobiles and commercial airplanes, Journal of Sound and Vibration 262 (2003) 457-474.

[5] E.A. Skelton and J.H. James, Acoustics of an anisotropic layered cylinder, Journal of Sound and Vibration 161(2) (1993), $251-264$.

[6] J.H. Lee and J. Kim, Sound transmission through periodically stiffened cylindrical shell, Journal of Sound and Vibration 251(3) (2002), $431-456$.

[7] J.H. Lee and J. Kim, Analysis and measurement of sound transmission through a double-walled cylindrical shell, Journal of Sound and Vibration 251(4) (2002), 631-649.

[8] J.W. Dickey, D.A. Nixon and J.M. D'Archangelo, Acoustic high-frequency scattering by elastic cylindrical shells, Journal of the Acoustical Society of America 74(1) (1983), 294-304. 
[9] J.S. Sastry and M.L. Munjal, Response of a multi-layered infinite cylinder to a plane wave excitation by means of transfer matrices, Journal of Sound and Vibration 209(1) (1998), 99-121.

[10] J.S. Sastry and M.L. Munjal, Response of a multi-layered infinite cylinder to two-dimensional pressure excitation by means of transfer matrices, Journal of Sound and Vibration 209(1) (1998), 123-142.

[11] J. Zemanek and I. Rudnick, Attenuation and dispersion of elastic waves in cylindrical bar, Journal of the Acoustical Society of America 33 (1961), 1283-1288.

[12] L. Cheng, Fluid-structural coupling of a plate-ended cylindrical shell: vibration and internal sound field, Journal of Sound and Vibration 174(5) (1994), 641-654.

[13] L. Flax and W.G. Neubauer, Acoustic reflection from layered elastic absorptive cylinders, Journal of the Acoustical Society of America 61(2) (1977), 307-312

[14] L. Flax, L.R. Dragonette and H. Uberall, Theory of elastic resonance excitation by sound scattering , Journal of the Acoustical Society of America 63(3) (1978), 723-730.

[15] L.R. Koval, On sound transmission into a thin cylindrical shell under flight conditions, Journal of Sound and Vibration 48(2) (1976), $265-275$.

[16] L.R. Koval, Effects of cavity resonances on sound transmission into a thin cylindrical shell, Journal of Sound and Vibration 59(1) (1978), 23-33.

[17] L.R. Koval, Sound transmission into a laminated composite cylindrical shell, Journal of Sound and Vibration 71(4) (1980), 523-530.

[18] M.C. Junger and J.M. Garrelick, Short-wave length back scattering cross sections of rigid and partially coated cylinders and spheres, Journal of the Acoustical Society of America 56(5) (1974), 1347-1360.

[19] P.H. White, Sound transmission through a finite, closed, cylindrical shell, Journal of the Acoustical Society of America 40(5) (1966), 1124-1130.

[20] P.M, Morse and K.U, Ingard, Theoretical Acoustics, 1968 New York McGraw-Hill.

[21] P.S. Dubbelday, Poisson's ratio of foamed aluminum determined by laser vibrometry, Journal of the Acoustical Society of America 91 (1992), 1737-1744.

[22] R.D. Doolittle and H. Uberall, Sound scattering by elastic cylindrical shells, Journal of the Acoustical Society of America 39(3) (1966), 272-275.

[23] S.M. Hasheminejad and N. Safari, Acoustic scattering from viscoelastically coated spheres and cylinders in viscous fluids, Journal of Sound and Vibration 280 (2005), 101-125.

[24] S.M. Hasheminejad and N. Safari, Dynamic viscoelastic effects on sound wave diffraction by spherical and cylindrical shells submerged in and filled with viscous compressible fluids, Shock and Vibration 10(5-6) (2003).

[25] S. Sim and K.J. Kim, A method to determine the complex modulus and Poisson's ratio of viscoelastic materials for FEM applications, Journal of Sound and Vibration 141 (1990), 71-82.

[26] T. Pritz, Measurement methods of complex Poisson's ratio of viscoelastic materials, Applied Acoustics 60 (2000), $279-292$.

[27] T. Pritz, Frequency power law of material damping, Applied Acoustics 65 (2004), 1027-1036.

[28] X.L. Bao, H. Cao and H. Uberal, Resonances and surface waves in the scattering of an obliquely incident acoustic field by an infinite elastic cylinder, Journal of the Acoustical Society of America 87(1) (1990), 106-110. 

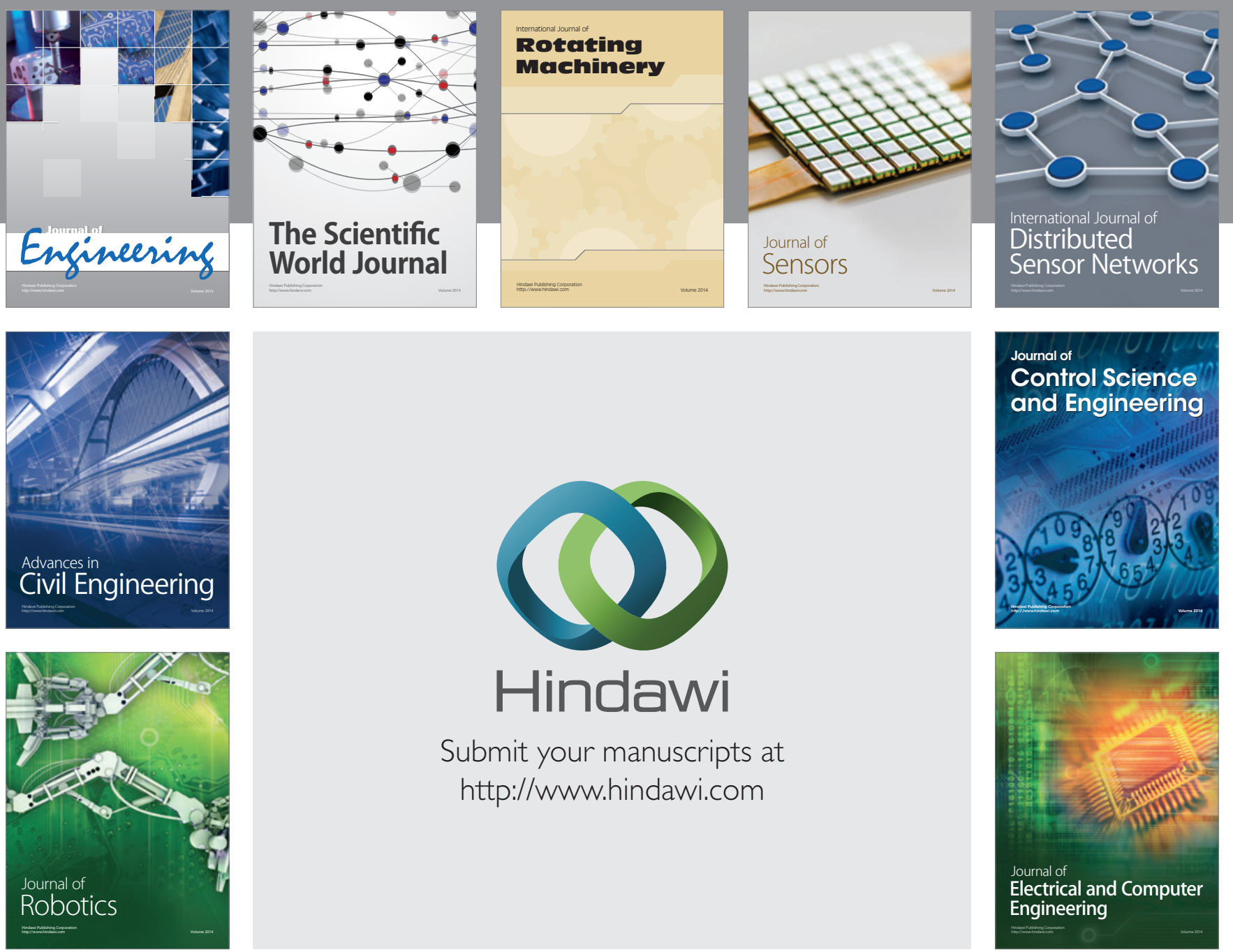

Submit your manuscripts at

http://www.hindawi.com
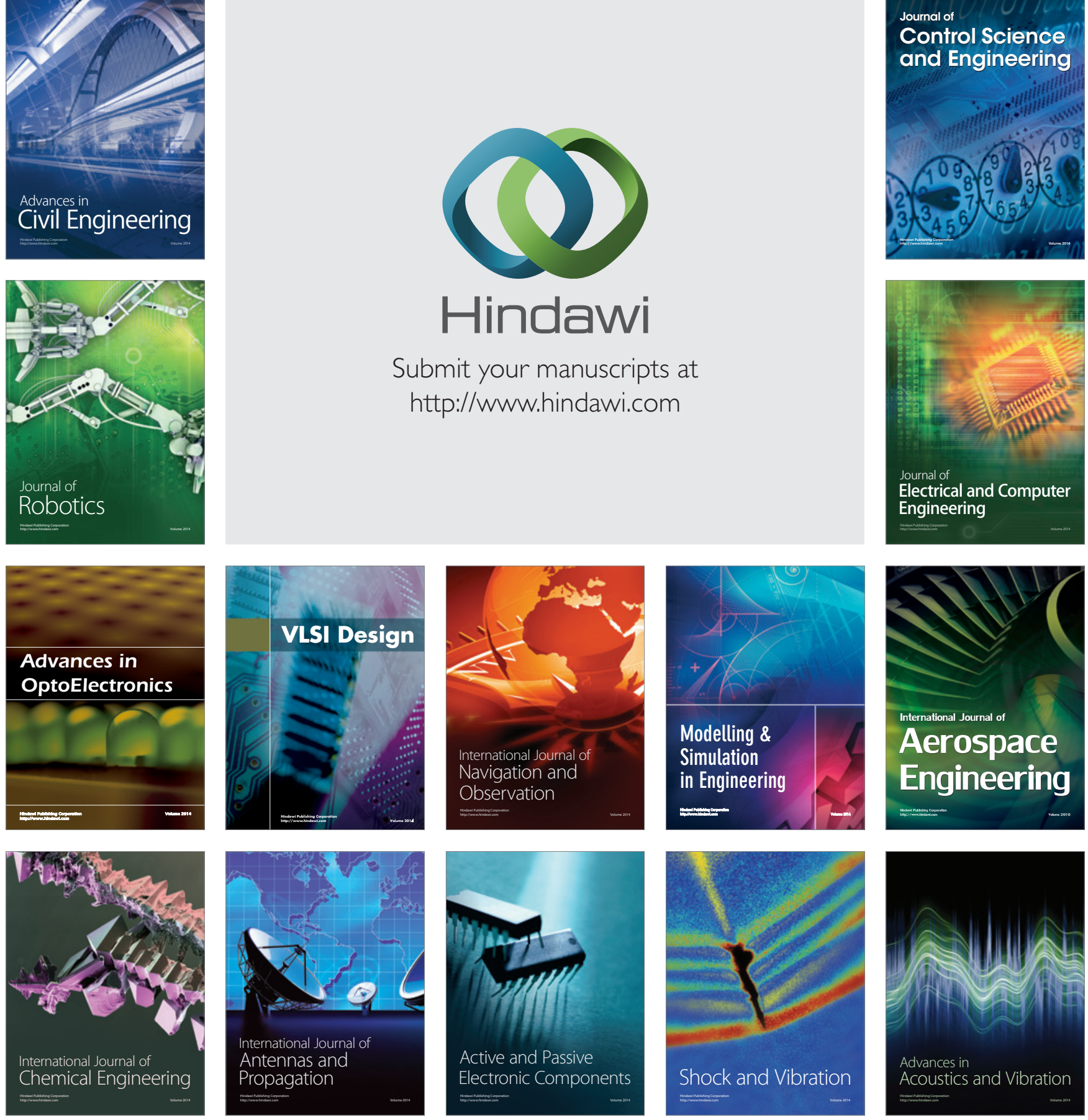